\title{
Quantum Systems Correlated with a Finite Bath: Nonequilibrium Dynamics and Thermodynamics
}

\author{
Andreu Riera-Campeny®, ${ }^{1,{ }^{*}}$ Anna Sanpera, ${ }^{1,2}$ and Philipp Strasberg ${ }^{1}$ \\ ${ }^{1}$ Física Teòrica: Informació i Fenòmens Quàntics, Departament de Física, Universitat Autònoma de Barcelona, \\ 08193 Bellaterra, Spain \\ ${ }^{2}$ ICREA, Passeig Lluís Companys 23, 08001 Barcelona, Spain
}

(Received 16 September 2020; revised 18 December 2020; accepted 12 January 2021; published 8 March 2021)

\begin{abstract}
Describing open quantum systems far from equilibrium is challenging, in particular when the environment is mesoscopic, when it develops nonequilibrium features during the evolution, or when memory effects cannot be disregarded. Here we derive a master equation that explicitly accounts for system-bath correlations and includes, at a coarse-grained level, a dynamically evolving bath. It applies to a wide variety of environments; for instance, those that can be described by random matrix theory or the eigenstate thermalization hypothesis. We obtain a local detailed balance condition that does not forbid the emergence of stable negative temperature states in unison with the definition of temperature through the Boltzmann entropy. We benchmark the master equation against the exact evolution and observe very good agreement in a situation where the conventional Born-Markov-secular master equation breaks down. The present description of the dynamics is robust and it remains accurate even if some of the assumptions are relaxed. Even though our master equation describes a dynamically evolving bath not described by a Gibbs state, we provide a consistent nonequilibrium thermodynamic framework and derive the first and second law as well as the Clausius inequality. Our work paves the way for studying a variety of nanoscale quantum technologies, including engines, refrigerators, and heat pumps, beyond the conventionally used assumption of a static thermal bath.
\end{abstract}

DOI: 10.1103/PRXQuantum.2.010340

\section{INTRODUCTION}

To understand the potential of future quantum technologies, it is essential to develop an efficient description of microscopic systems far from equilibrium. Quantum master equations are an important tool to describe the nonequilibrium dynamics of small systems in contact with an external environment [1-3]. Master equations have the advantage that they apply to a large class of open systems, are intuitive, and often allow further analytical progress in the description. Unfortunately, master equations often rely on the assumption that the environment is large, thermal, memoryless, and weakly coupled to the system; therefore, they quickly break down for many interesting applications [1-3].

Here we reconsider a class of master equations, first proposed in Ref. [4], which are general, intuitive, and

\footnotetext{
*andreu.riera.campeny@uab.cat
}

Published by the American Physical Society under the terms of the Creative Commons Attribution 4.0 International license. Further distribution of this work must maintain attribution to the author(s) and the published article's title, journal citation, and DOI. analytically tractable but overcome to some extent the assumption of a large, thermal, and memoryless environment. We refer to them as the "extended microcanonical master equation" (EMME). The idea is to additionally keep track of the bath dynamics at a coarse-grained level and include to some degree system-bath correlations. This approach was previously formalized using correlated projection operator techniques [5-8] and it has been shown to significantly improve standard perturbative master equations [6,8-10]. However, it has not yet become a widespread tool. We believe the reason is that the general physical properties of this class of master equations have not yet been investigated and applications have remained restricted to specifically tailored models. It is our goal to overcome these limitations in the present paper.

We show that the EMME does not only provide an efficient way to describe the non-Markovian dynamics of open quantum systems but it also connects to a plethora of actively discussed topics in nonequilibrium statistical mechanics. In the following, we summarize our main results, which also serves as an outline for the rest of the paper.

In Sec. II, we derive the EMME using three different methods, all leading to the same structure and 
phenomenology. Among them, one method uses random matrix theory (RMT) and another invokes the eigenstate thermalization hypothesis (ETH). The fact that we obtain the same equation using different methods indicates that our master equation has a clear degree of universility since, in principle, it can be applied to many open quantum systems. In Sec. III, we observe that the EMME preserves the total (system plus bath) coarse-grained energy and we derive the local detailed balance. Remarkably, the local detailed balance condition does not forbid the emergence of stable negative temperatures defined according to the Boltzmann entropy. We devote Sec. IV to testing numerically our analytical results. We benchmark the EMME against the frequently used Born-Markov-secular (BMS) master equation and against the exact dynamics. To that end, we consider a spin system randomly coupled to a finite environment for which the EMME shows very good agreement with the exact dynamics. In Sec. V, we introduce a consistent nonequilibrium thermodynamic framework that includes slowly driven systems. Using this framework, we obtain the first and second law of thermodynamics. Moreover, we connect the first and second law with the Clausius inequality by introducing an effective nonequilibrium temperature. In Sec. VI, we extend the aforementioned results to the case of multiple environments. In Sec. VII, we compare the EMME with other master equation approaches and present our conclusions. Finally, to keep the presentation focused, generalizations and additional results are shifted to the Appendixes.

\section{THE EXTENDED MICROCANONICAL MASTER EQUATION}

\section{A. General idea and final result}

One of the central goals of the theory of open quantum systems is to derive a closed evolution equation for the relevant degrees of freedom. Such an equation can be formally obtained with use of projection operator techniques $[1,3]$. Projection operator techniques are based on the definition of a projection superoperator $\mathcal{P}$ and its complementary $\mathcal{Q}=\mathcal{I}-\mathcal{P}$ (where $\mathcal{I}$ is the identity map), which divide the Hilbert space into relevant $(\mathcal{P})$ and irrelevant $(\mathcal{Q})$ degrees of freedom. Because $\mathcal{P}$ and $\mathcal{Q}$ are orthogonal projectors, they satisfy $\mathcal{P}^{2}=\mathcal{P}, \mathcal{Q}^{2}=\mathcal{Q}$, and $\mathcal{Q P}=\mathcal{P} \mathcal{Q}=0$, and are otherwise quite arbitrary. Given the state $\rho$ of an isolated system, the use of projection techniques provides a closed equation for the dynamics of the relevant part $\mathcal{P} \rho=\mathcal{P}[\rho]$, achieved by formally integrating out the dynamics of the irrelevant part $\mathcal{Q}[\rho][1,3]$.

We consider an isolated system (the universe) composed of the system $\mathrm{S}$ and the environment (or bath) B. The isolated system undergoes unitary dynamics generated by the Hamiltonian $\mathrm{H}=\mathrm{H}_{\mathrm{S}}+\mathrm{H}_{\mathrm{int}}+\mathrm{H}_{\mathrm{B}}$, where $\mathrm{H}_{\mathrm{S}}$ and $\mathrm{H}_{\mathrm{B}}$ contain only system and bath degrees of freedom, respectively, while $\mathrm{H}_{\text {int }}$ represents the interaction energy between the system and the bath.

In the interaction picture with respect to $\mathrm{H}_{0}=\mathrm{H}_{\mathrm{S}}+\mathrm{H}_{B}$, the evolution of the isolated system is generated by the von Neumann equation $(\hbar=1)$

$$
\partial_{t} \tilde{\rho}(t)=-i\left[\tilde{\mathrm{H}}_{\mathrm{int}}(t), \tilde{\rho}(t)\right]:=\mathcal{L}(t)[\tilde{\rho}(t)]
$$

where the tilde denotes operators in the interaction picture; for example, $\tilde{\rho}(t)=\exp \left(i \mathrm{H}_{0} t\right) \rho(0) \exp \left(-i \mathrm{H}_{0} t\right)$. Under the assumptions of (i) weak coupling and (ii) an initial state contained in the relevant part $\mathcal{P}[\rho(0)]=\rho(0)$, the dynamics of the relevant degrees of freedom are described by the well-known second-order time-convolutionless (or finitetime Redfield) master equation $[1,3]$ :

$$
\begin{aligned}
\partial_{t} \mathcal{P} \tilde{\rho}(t)= & \mathcal{P} \mathcal{L}(t)[\mathcal{P} \tilde{\rho}(t)] \\
& +\int_{0}^{t} d t^{\prime} \mathcal{P} \mathcal{L}(t) \mathcal{Q L}\left(t^{\prime}\right)[\mathcal{P} \tilde{\rho}(t)],
\end{aligned}
$$

where we have disregarded terms of $\mathcal{O}\left(\mathrm{H}_{\text {int }}^{3}\right)$. Dropping assumption (ii) would lead to an extra nonhomogeneous term in Eq. (2) that, typically, is relevant only for the transient dynamics.

It is worth noting that the derivation of Eq. (2) makes no use of the explicit form of the projection superoperator $\mathcal{P}$. For later comparison, we introduce the projection superoperator $\mathcal{P}_{\text {Born }}$, which leads to the standard BMS master equation $\left(k_{\mathrm{B}}=1\right)$ :

$$
\mathcal{P}_{\text {Born }}[\rho]:=\rho_{\mathrm{S}} \otimes \frac{e^{-\mathrm{H}_{\mathrm{B}} / T_{\mathrm{can}}}}{Z_{\mathrm{B}}},
$$

where $\rho_{\mathrm{S}}:=\operatorname{tr}_{\mathrm{B}} \rho, T_{\text {can }}$ is the canonical temperature of the reference state of the bath, and $Z_{\mathrm{B}}:=\operatorname{tr}\left[\exp \left(-\mathrm{H}_{\mathrm{B}} / T_{\text {can }}\right)\right]$ is the partition function. We emphasize that $\mathcal{P}_{\text {Born }}$ is defined with respect to a fixed Gibbs state of the bath, which is uncorrelated with the system.

In some physical situations, however, the system-bath interaction causes the bath to evolve and develop correlations with the system. To better approximate this situation, we instead consider the following classically correlated projection superoperator:

$$
\mathcal{P}[\rho]:=\sum_{E} \rho_{\mathrm{S}}(E) \otimes \frac{\Pi_{E}}{V_{E}},
$$

where all the terms deserve a comment. First, the macroscopic energies $\{E\}$ are a set of coarse-grained bath energies. To be precise, consider the spectral decomposition of the bath Hamiltonian $\mathrm{H}_{\mathrm{B}}=\sum_{E_{i}} E_{i}\left|E_{i}\right\rangle\left\langle E_{i}\right|$, where the set of microscopic energies $\left\{E_{i}\right\}$ is ordered according to $E_{i} \leq E_{j}$ if $i<j$. In contrast, we define the set $\{E\}$ of macroscopic energies by dividing the spectrum of the bath 
into nonoverlapping energy windows $E_{\delta}:=[E-\delta / 2, E+$ $\delta / 2$ ) of width $\delta$. Accordingly, we introduce the projectors $\Pi_{E}:=\sum_{E_{i} \in E_{\delta}}\left|E_{i}\right\rangle\left\langle E_{i}\right|$ corresponding to the different energies $E$ that can be distinguished by macroscopic measurements. We also introduce the volume $V_{E}:=\operatorname{tr} \Pi_{E}$, which represents the number of microstates in the macrostate $E$. Finally, $\rho_{\mathrm{S}}(E):=\operatorname{tr}_{\mathrm{B}}\left(\rho \Pi_{E}\right)$ is the unnormalized conditional state of the system when the bath is found in the macroscopic state $E$, and its trace gives the probability $p(E):=\operatorname{tr} \rho_{\mathrm{S}}(E)$ of the bath being in that macrostate $E$. Then the reduced state of the system can be obtained as $\rho_{\mathrm{S}}=\sum_{E} \rho_{\mathrm{S}}(E)$, which is normalized since $\sum_{E} p(E)=1$. For the time being, we focus on the case where $\mathrm{H}_{\mathrm{B}}$ represents a single bath, leaving the extension to multiple environments to Sec. VI.

Our goal is to describe the dynamics of open quantum systems that interact and build up correlations with a finite bath. Hence, we first define precisely what a finite bath actually is. First, the term "finite" refers to an environment with a finite dimension whose state cannot be approximated by a time-independent reference state. Second, the term "bath" implies that such a system should exhibit bathlike properties, which are ultimately related to a large number of microstates. In particular, the coarse-graining procedure should ensure that in each energy window $E_{\delta}$ there are enough microscopic energies $E_{i}$. As already recognized by Boltzmann, the aforementioned coarsegraining procedure is crucial to reconcile the underlying reversible quantum mechanical description with the irreversible macroscopic world and permits a simplified dynamical description. The same coarse-graining procedure was also used by von Neumann [11] (see Ref. [12] for the English translation) almost a century ago.

To fix further notation, we introduce the system Hamiltonian $\mathrm{H}_{\mathrm{S}}=\sum_{k} \varepsilon_{k}|k\rangle\langle k|$ and we fix the interaction $\mathrm{H}_{\text {int }}=$ $\lambda S \otimes B_{\text {int }}$, where $\lambda$ is an energy scale. The general expressions for multiple coupling operators (i.e., $\mathrm{H}_{\text {int }}=\sum_{\alpha} S^{\alpha} \otimes$ $\left.B_{\text {int }}^{\alpha}\right)$ can be found in the Appendixes.

Under the conditions set out above, our central object of study is a master equation describing the time evolution of $\rho_{\mathrm{S}}(E)$. If we use the conventional Markov and secular approximations [1-3], it reads

$$
\begin{aligned}
\partial_{t} \rho_{\mathrm{S}}(E)= & -i\left[\mathrm{H}_{\mathrm{S}}^{\prime}(E), \rho_{\mathrm{S}}(E)\right] \\
& +\sum_{\omega}\left(\frac{\gamma(E, E-\omega)}{V_{E-\omega}} S_{\omega} \rho_{\mathrm{S}}(E-\omega) S_{\omega}^{\dagger}\right. \\
& \left.-\frac{\gamma(E+\omega, E)}{2 V_{E}}\left\{\rho_{\mathrm{S}}(E), S_{\omega}^{\dagger} S_{\omega}\right\}\right),
\end{aligned}
$$

where $\omega$ sums over all possible system transition frequencies. Furthermore, we have introduced the dissipation rates

$$
\gamma\left(E, E^{\prime}\right) \delta_{E^{\prime}, E+\omega}:=\int_{\mathbb{R}} d \tau \operatorname{tr}_{\mathrm{B}}\left[\tilde{B}^{\dagger}(-\tau) \Pi_{E} B \Pi_{E^{\prime}}\right] e^{i \omega \tau},
$$

the operators $S_{\omega}:=\sum_{k q}\langle k|S| q\rangle|k\rangle\langle q| \delta_{\varepsilon_{q}-\varepsilon_{k}, \omega}$, and the modified Hamiltonian $\mathrm{H}_{\mathrm{S}}^{\prime}(E)$, which commutes with the bare system Hamiltonian $\mathrm{H}_{\mathrm{S}}$. All of them are defined precisely below.

It is important to emphasize two general features of our EMME. First, one can show that Eq. (5) fits into the general form investigated by Breuer [8], who shows that it preserves the trace of $\rho_{\mathrm{S}}$ and complete positivity of $\rho_{\mathrm{S}}(E)$ at all times. Second, although we derive Eq. (5) using the Markov and secular approximations, which implies that $\rho_{\mathrm{S}}(E)$ evolves in a Markovian manner, the reduced system state $\rho_{\mathrm{S}}$ does not. Therefore, the EMME is able to capture non-Markovian system dynamics.

We provide a step-by-step derivation of Eq. (5) in Sec. II B. Particular care is required when one is evaluating the bath correlation function. In Sec. II C, we use three different methods to arrive at the same conclusion. Further mathematical details are shifted to Appendix A. Readers not interested in the details of the derivation can skip the rest of this section and continue reading in Sec. III, where we start to focus in detail on the physics predicted by the EMME.

\section{B. Detailed derivation}

In this subsection, we give a detailed derivation of the EMME, which corresponds to finding explicit expressions for the first-order and second-order terms in Eq. (2). To this aim, it will prove useful to decompose $\mathrm{H}_{\text {int }}$ into blockdiagonal and off-diagonal parts:

$$
\mathrm{H}_{\mathrm{int}}=\sum_{E} \delta \mathrm{H}(E) \otimes \Pi_{E}+V,
$$

where we have implicitly defined

$$
\begin{aligned}
& \delta \mathrm{H}(E):=\lambda\left\langle B_{\mathrm{int}}\right\rangle_{E} S, \\
& V:=\lambda S \otimes B=\lambda S \otimes\left(B_{\mathrm{int}}-\sum_{E}\left\langle B_{\mathrm{int}}\right\rangle_{E} \Pi_{E}\right) .
\end{aligned}
$$

Here $\langle\cdots\rangle_{E}:=\operatorname{tr}_{\mathrm{B}}\left(\cdots \Pi_{E} / V_{E}\right)$ denotes the microcanonical average at energy $E$. Note that the operator $\mathrm{B}$ has the important property $\langle B\rangle_{E}=0$, which we use below.

With use of Eq. (7), the first-order term in Eq. (2) reads

$$
\mathcal{P} \mathcal{L}(t)[\mathcal{P} \tilde{\rho}(t)]=-i \sum_{E}\left[\delta \tilde{\mathrm{H}}(E ; t) \Pi_{E}, \mathcal{P} \tilde{\rho}(t)\right]
$$

In standard projection operator techniques that use $\mathcal{P}_{\text {Born, }}$ the first-order term in Eq. (2) can be set to zero without loss 
of generality by including its contribution in the system Hamiltonian $\mathrm{H}_{\mathrm{S}}$ [1-3]. This is no longer possible for the projection in Eq. (4). The second-order term is obtained by similar manipulations as

$$
\begin{aligned}
& \mathcal{P} \mathcal{L}(t) \mathcal{Q L}\left(t^{\prime}\right)[\mathcal{P} \tilde{\rho}(t)] \\
& \quad=-\sum_{E} \operatorname{tr}_{B}\left\{\Pi_{E}\left[\tilde{V}(t),\left[\tilde{V}\left(t^{\prime}\right), \mathcal{P} \tilde{\rho}(t)\right]\right]\right\} \otimes \frac{\Pi_{E}}{V_{E}} .
\end{aligned}
$$

The evolution equation for each component $\tilde{\rho}_{\mathrm{S}}(E ; t)$ is then found by inserting Eqs. (9) and (10) in Eq. (2) and using of our correlated projector $\mathcal{P}$ in Eq. (4). This yields

$$
\begin{aligned}
& \partial_{t} \tilde{\rho}_{\mathrm{S}}(E ; t)=-i\left[\delta \tilde{\mathrm{H}}(E ; t), \tilde{\rho}_{\mathrm{S}}(E ; t)\right] \\
& \quad+\sum_{E^{\prime}} \int_{0}^{t} d t^{\prime} \operatorname{tr}_{\mathrm{B}}\left\{\Pi_{E}\left[\tilde{V}(t),\left[\tilde{\rho}_{\mathrm{S}}\left(E^{\prime} ; t\right) \otimes \frac{\Pi_{E^{\prime}}}{V_{E^{\prime}}}, \tilde{V}\left(t^{\prime}\right)\right]\right]\right\} .
\end{aligned}
$$

Next, we introduce the microcanonical bath correlation function

$$
C_{\mathrm{B}}\left(E, E^{\prime} ; t^{\prime}-t\right):=\lambda^{2} \operatorname{tr}\left\langle B^{\dagger}\left(t^{\prime}\right) \Pi_{E} B(t)\right\rangle_{E^{\prime}},
$$

which can be explicitly computed as

$$
C_{\mathrm{B}}\left(E, E^{\prime} ;-\tau\right)=\sum_{E_{i} \in E_{\delta}} \sum_{E_{j} \in E_{\delta}^{\prime}} \frac{\lambda^{2}}{V_{E^{\prime}}}\left|\left\langle E_{i}|B| E_{j}\right\rangle\right|^{2} e^{i\left(E_{i}-E_{j}\right) \tau} .
$$

We also introduce the decomposition $\tilde{S}(t)=\sum_{\omega} S_{\omega}$ $\exp (-i \omega t)$, where $S_{\omega}:=\sum_{k q}\langle k|S| q\rangle|k\rangle\langle q| \delta_{\varepsilon_{q}-\varepsilon_{k}, \omega}$. Using both expressions in Eq. (11), one arrives at

$$
\begin{aligned}
& \partial_{t} \tilde{\rho}_{\mathrm{S}}(E ; t)=-i\left[\tilde{\mathrm{H}}(E ; t), \tilde{\rho}_{\mathrm{S}}(E ; t)\right] \\
& \quad+\sum_{E^{\prime}} \sum_{\omega \omega^{\prime}} \int_{0}^{t} d t^{\prime} e^{i\left(\omega^{\prime} t^{\prime}-\omega t\right)}\left(C_{\mathrm{B}}\left(E, E^{\prime} ; t^{\prime}-t\right) S_{\omega} \tilde{\rho}_{\mathrm{S}}\left(E^{\prime}, t\right) S_{\omega^{\prime}}^{\dagger}\right. \\
& \left.\quad-C_{\mathrm{B}}\left(E^{\prime}, E ; t^{\prime}-t\right) \tilde{\rho}_{\mathrm{S}}(E ; t) S_{\omega^{\prime}}^{\dagger} S_{\omega}\right)+ \text { h.c. }
\end{aligned}
$$

Equation (14) is the finite-time Redfield version of the EMME (in the interaction picture), which is ready for numerical implementation and gives improved results for transient times (see Sec. IV). It is, however, still hard to work with Eq. (14) analytically. Therefore, we use the standard Markov and secular approximations [1-3], which, nonetheless, give different results from the standard BMS master equation due to the different choice of the projection superoperator $\mathcal{P}$ in Eq. (4).

The Markov approximation relies on the fact that the microcanonical bath correlation function decays rapidly to zero. To understand the range of validity of this approximation, we use the following timescale argument. We denote by $\tau_{\mathrm{B}}$ the correlation time of the bath defined such that $C_{\mathrm{B}}\left(E, E^{\prime} ;-\tau\right) \approx 0$ for all $\tau \geq \tau_{\mathrm{B}}$. Of course, $\tau_{\mathrm{B}}$ is a function of the energy width $\delta$, that is, $\tau_{\mathrm{B}}=\tau_{\mathrm{B}}(\delta)$, and depends on the particular coarse-graining procedure. If the bath energies are fine-grained (i.e., $\delta \rightarrow 0$ ), the correlation function oscillates at frequency $E_{i}-E_{j}$ and never decays. In such a case, $\tau_{\mathrm{B}} \rightarrow \infty$ and the Markov approximation breaks down. Instead, for a sufficiently large $\delta$ many frequencies $E_{i}-E_{j}$ contribute to Eq. (13) and the correlation function decays rapidly. Then one can safely extend the upper limit of the time integrals in Eq. (14) to infinity and the Markov approximation holds. In this sense, a finite coarse graining $\delta$ is necessary to reconcile the reversible microscopic description with the irreversible macroscopic world.

However, for finite baths the correlation function never decays exactly to zero and it can exhibit recurrences for sufficiently long times. Then the validity of the Markov assumption relies on the fact that the typical Poincare recurrence time is exceedingly large (see, for instance, Refs. $[13,14])$, and one is often interested in timescales of evolution much smaller than the recurrence time.

Our second approximation, which is called the "secular approximation", consists in averaging out the rapidly oscillating terms in the interaction picture. Formally, this is done by introducing the time average

$$
\bar{O}:=\lim _{T \rightarrow \infty} \frac{1}{T} \int_{0}^{T} d t^{\prime} \tilde{O}\left(t^{\prime}\right)
$$

which, used in Eq. (14) (after taking the limit $t \rightarrow \infty$ in the upper limit of the integral) selects the components $\omega=\omega^{\prime}$. Introducing the one-sided Fourier transform

$$
\Gamma\left(E, E^{\prime} ; \omega\right):=V_{E^{\prime}} \int_{0}^{\infty} d \tau C_{\mathrm{B}}\left(E, E^{\prime} ;-\tau\right) e^{i \omega \tau},
$$

we can write the resulting equation after both approximations compactly as

$$
\begin{aligned}
\partial_{t} \tilde{\rho}_{\mathrm{S}}(E ; t)= & -i\left[\overline{\delta \mathrm{H}}(E), \tilde{\rho}_{\mathrm{S}}(E ; t)\right] \\
& +\sum_{E^{\prime}} \sum_{\omega}\left(\frac{\Gamma\left(E, E^{\prime} ;-\omega\right)}{V_{E^{\prime}}} S_{\omega} \tilde{\rho}_{\mathrm{S}}\left(E^{\prime} ; t\right) S_{\omega}^{\dagger}\right. \\
& \left.-\frac{\Gamma\left(E^{\prime}, E ;-\omega\right)}{V_{E}} \tilde{\rho}_{\mathrm{S}}(E ; t) S_{\omega}^{\dagger} S_{\omega}\right)+ \text { h.c. },
\end{aligned}
$$

where the change of variables $\tau=t-t^{\prime}$ has been performed. Also, $\overline{\delta \mathrm{H}}(E)$ is an energy-dependent Hamiltonian shift that commutes with the system Hamiltonian $\mathrm{H}_{\mathrm{S}}$ and has the explicit expression $\overline{\delta \mathrm{H}}(E)=$ $\sum_{k}\left\langle B_{\text {int }}\right\rangle_{E}\langle k|S| k\rangle|k\rangle\langle k|$.

To compare the EMME with the conventional BMS master equation, it is convenient to decompose the function 
$\Gamma\left(E, E^{\prime} ; \omega\right)$ into its real and imaginary parts as

$$
\begin{aligned}
& \mathrm{A}\left(E, E^{\prime} ; \omega\right):=\frac{1}{2 i}\left[\Gamma\left(E, E^{\prime} ; \omega\right)-\Gamma\left(E, E^{\prime} ; \omega\right)^{*}\right], \\
& \gamma\left(E, E^{\prime} ; \omega\right):=\Gamma\left(E, E^{\prime} ; \omega\right)+\Gamma\left(E, E^{\prime} ; \omega\right)^{*},
\end{aligned}
$$

from which it follows that

$$
\gamma\left(E, E^{\prime} ; \omega\right)=V_{E^{\prime}} \int_{\mathbb{R}} d \tau C_{\mathrm{B}}\left(E, E^{\prime} ;-\tau\right) e^{i \omega \tau} .
$$

From Sec. II C 1 to Sec. II C 3, we show that the function $\gamma\left(E, E^{\prime} ; \omega\right)$ is generically peaked around $E^{\prime}=E+\omega$ and we are allowed to factorize

$$
\gamma\left(E, E^{\prime} ; \omega\right)=\gamma\left(E, E^{\prime}\right) \delta_{E^{\prime}, E+\omega} .
$$

We also introduce the energy-dependent Lamb-shift Hamiltonian

$$
\mathrm{H}_{\mathrm{LS}}(E):=-\sum_{E^{\prime}} \sum_{\omega} \frac{\mathrm{A}\left(E^{\prime}, E,-\omega\right)}{V_{E}} S_{\omega}^{\dagger} S_{\omega},
$$

which also commutes with the system Hamiltonian $\mathrm{H}_{\mathrm{S}}$. Then the modified Hamiltonian

$$
\mathrm{H}_{\mathrm{S}}^{\prime}(E):=\mathrm{H}_{\mathrm{S}}+\overline{\delta \mathrm{H}}(E)+\mathrm{H}_{\mathrm{LS}}(E)
$$

commutes with $\mathrm{H}_{\mathrm{S}}$ and corresponds to an $E$-dependent shift of the system energies.

Finally, after moving to the Schrödinger picture, we obtain our first main result: the EMME within the Markov and secular approximations shown in Eq. (5).

The exact computation of $C_{\mathrm{B}}\left(E, E^{\prime} ;-\tau\right)$, or the associated $\gamma\left(E, E^{\prime} ; \omega\right)$, depends on the fine structure of the bath energy levels as well as the exact form of the coupling operators B. In relevant physical situations, neither the fine structure of the bath energy levels nor the exact form of the coupling operator is typically available. For this reason, it is important to find approximate methods to compute the correlation function that depend only on the coarse structure of the bath energy levels. In the following subsection, we present three methods to obtain the functions $\gamma\left(E, E^{\prime} ; \omega\right)$ : the first one ignores part of the internal bath dynamics, the second one uses RMT, and the third invokes the ETH. Moreover, we further connect those three methods in Appendix A 4.

\section{Evaluation of the bath correlation function}

\section{Heuristic approach}

The idea behind the heuristic approach is to assume that there exists a coarse graining $\delta$ such that $\delta \tau_{\mathrm{B}}(\delta) \ll 1$. In that case, for the relevant times $\tau \leq \tau_{\mathrm{B}}$, one can expand

$$
e^{i\left(E_{i}-E_{j}\right) \tau} \approx e^{i\left(E-E^{\prime}\right) \tau}+\mathcal{O}\left(\delta \tau_{\mathrm{B}}\right) .
$$

Essentially, this corresponds to replacing the energy differences $E_{i}-E_{j} \mapsto E-E^{\prime}$ when $E_{i} \in E_{\delta}$ and $E_{j} \in E_{\delta}^{\prime}$ in Eq.
(13), as was considered in Ref. [15]. Then the correlation function yields

$$
C_{\mathrm{B}}\left(E, E^{\prime} ;-\tau\right) \approx \lambda^{2} \operatorname{tr}\left(B^{\dagger} \Pi_{E} B \Pi_{E^{\prime}}\right) \frac{e^{i\left(E-E^{\prime}\right) \tau}}{V_{E^{\prime}}}
$$

The case $\delta \rightarrow 0$ was studied in Refs. [4,16]. With the help of Eqs. (19) and (24) (see Appendix A 1), one obtains the dissipation rates

$$
\gamma_{\text {heuristic }}\left(E, E^{\prime}\right)=\frac{2 \pi \lambda^{2}}{\delta} \operatorname{tr}\left(B^{\dagger} \Pi_{E} B \Pi_{E^{\prime}}\right)
$$

Hence, the procedure above provides an additional interpretation of the parameter $\delta$. Of course, given a bath, it is not clear whether such a coarse graining $\delta$ exists, and for this reason we refer to this approach as "heuristic". On the other hand, the present evaluation of the correlation function does not rely on any explicit assumption on the bath coupling operator B. Alternative methods to evaluate $C_{\mathrm{B}}\left(E, E^{\prime} ;-\tau\right)$ that are based on assumptions about the bath coupling operator $\mathrm{B}$ are considered in the next two subsections.

\section{Random matrix coupling with a dense environment}

In many physical situations, the interaction Hamiltonian $\mathrm{H}_{\text {int }}$ might be too complicated to be obtained with $a b$ initio methods. Hence, in the same spirit as the heuristic approach, our aim is to evaluate the correlation function without fully specifying the bath coupling operator B. One possibility is offered by RMT (for a review of the topic see, for instance, Ref. [17]), which has been widely used in many physical contexts due to its universality. In particular, it has been used to describe the decay of quantum systems in contact with complex environments (see for instance, Refs. [6,9,18-23]). In general, a random matrix ansatz seems to work well for strongly nonintegrable systems (see, for instance, Ref. [24]).

Our approach is based on extracting B from a random matrix ensemble and computing the corresponding correlation function. In principle, two different members of the ensemble can give rise to a very different dynamics. The essence of RMT relies on the fact that this is often not the case and the fine structure of the coupling operators is important only in exceptional cases. In particular, it has been shown not only that the RMT approach gives the correct mean value when compared with the predictions of statistical mechanics but also that the variance between the two is very small (see, for instance, Ref. [25]). Thus, almost all members of the random matrix ensemble give the same prediction. Hence, despite our performing the random matrix ensemble average theoretically, no such average is implied experimentally. 
Here we use the RMT approach and consider that the coupling to the environment is done via the random matrix

$$
B=\sum_{E \neq E^{\prime}} \sum_{E_{i} \in E_{\delta}} \sum_{E_{j} \in E_{\delta}^{\prime}}\left[b\left(E, E^{\prime}\right)+c\left(E_{i}, E_{j}\right)\right]\left|E_{i}\right\rangle\left\langle E_{j}\right|,
$$

where $b\left(E, E^{\prime}\right)$ are deterministic functions of the macroscopic energies, and $c\left(E_{i}, E_{j}\right)$ are random numbers. We consider $c\left(E_{i}, E_{j}\right)$ to be independent and identically distributed complex random variables with zero mean, that is, $\mathbb{E}\left[c\left(E_{i}, E_{j}\right)\right]=0$, and variance $a^{2}$, that is, $\mathbb{E}\left[c\left(E_{i}, E_{j}\right) c\left(E_{i^{\prime}}, E_{j^{\prime}}\right)\right]=a^{2} \delta_{E_{i}, E_{i}^{\prime}} \delta_{E_{j}, E_{j}^{\prime}}$. Using the random coupling of Eq. (26) and averaging over the correlation function in Eq. (13) (see Appendix A 2 for details), one obtains the dissipation rates

$$
\gamma_{\mathrm{RMT}}\left(E, E^{\prime}\right)=\frac{2 \pi \lambda^{2}}{\delta} V_{E} V_{E^{\prime}}\left(\left|b\left(E, E^{\prime}\right)\right|^{2}+a^{2}\right) .
$$

\section{The eigenstate thermalization hypothesis}

The ETH is an ansatz for the matrix elements of a local observable in the energy eigenbasis of a quantum manybody system (see Refs. [26-29]). It has been successfully used to study equilibration and thermalization in a variety of isolated quantum systems. Yet, its exact range of validity is still under debate, but there is a consensus that it applies to many-body systems whose classical counterpart is chaotic (although not exclussively, see Ref. [28]). Here we use the ETH to make progress in computing the bath correlation function $C_{\mathrm{B}}\left(E, E^{\prime} ;-\tau\right)$, thereby linking the field of equilibration and thermalization in isolated many-body systems to the field of open quantum systems. The ETH can be formulated as follows. The matrix elements of a local observable $O$ in the energy eigenbasis of a nonintegrable quantum many-body system obey the following ansatz:

$$
O_{i j}=O\left(E_{i j}\right) \delta_{i j}+\sqrt{\frac{1}{V_{E_{i j}}}} f\left(E_{i j}, \Omega_{i j}\right) R_{i j},
$$

where the mean energy $E_{i j}=\left(E_{i}+E_{j}\right) / 2$ and the energy difference $\Omega_{i j}=E_{i}-E_{j}$ have been introduced. All elements of the above equation deserve a comment. The functions $O\left(E_{i j}\right)$ and $f\left(E_{i j}, \Omega_{i j}\right)$ are smooth functions of their arguments. Moreover, the function $f$ must decay as $\left|\Omega_{i j}\right|$ grows and has the symmetry property $f\left(E_{i j},-\Omega_{i j}\right)=$ $f^{*}\left(E_{i j}, \Omega_{i j}\right)$. Finally, the numbers $R_{i j}=R_{j i}^{*}$ have zero mean and unit variance, and vary erratically with $i$ and $j$. These erratically varying $R_{i j}$ allow us to effectively use arguments from random matrix theory without the need to actually perform any ensemble average.

The main insight arises from the fact that the open system $\mathrm{S}$ couples locally (through its boundary) to the bath $B$ via the operators B. Since the ETH holds for local observables of a quantum many-body system, we can make progress on the computation of the bath correlation function $C_{\mathrm{B}}\left(E, E^{\prime} ;-\tau\right)$ using the ETH ansatz for the operators B. Then, introducing $\bar{E}=\left(E+E^{\prime}\right) / 2$, one arrives at the complex dissipation rates (see Appendix A 3)

$$
\gamma_{\mathrm{ETH}}\left(E, E^{\prime}\right)=\frac{2 \pi \lambda^{2}}{\delta} V_{E} V_{E^{\prime}} \frac{\left|f\left(\bar{E}, E-E^{\prime}\right)\right|^{2}}{V_{\bar{E}}} .
$$

\section{ENERGY CONSERVATION, EQUILIBRIUM STATES, AND MUTUAL INFORMATION}

After proving that the EMME has a certain degree of universality, we devote this section to investigating its properties. We start by noting that the populations $p\left(\varepsilon_{k}, E\right)=\left\langle k\left|\rho_{\mathrm{S}}(E)\right| k\right\rangle$ evolve autonomously under the rate equation

$$
\begin{aligned}
\partial_{t} p\left(\varepsilon_{k}, E\right)= & \sum_{q}\left(\frac{W_{k q}\left(E, E+\omega_{k q}\right)}{V_{E+\omega_{k q}}} p\left(\varepsilon_{q}, E+\omega_{k q}\right)\right. \\
& \left.-\frac{W_{q k}\left(E+\omega_{k q}, E\right)}{V_{E}} p\left(\varepsilon_{k}, E\right)\right),
\end{aligned}
$$

where we have defined $\omega_{k q}=\varepsilon_{k}-\varepsilon_{q}$ and the transition rates

$$
W_{k q}\left(E, E^{\prime}\right):=\gamma\left(E, E^{\prime}\right)|\langle k|S| q\rangle|^{2} .
$$

In Appendix $C$, we prove the positivity of the transition rates $W_{k q}\left(E, E^{\prime}\right) \geq 0$ as well as the symmetry $W_{k q}\left(E, E^{\prime}\right)=$ $W_{q k}\left(E^{\prime}, E\right)$.

\section{A. Strict energy conservation}

A crucial property of Eq. (5) is that the coarse-grained total energy of the system and bath composite is preserved under the evolution. The statement is actually stronger since not only the average value of the total energy is preserved but also the associated probability distribution. To be precise, we introduce the coarse-grained total energy $E_{\text {tot }}:=\varepsilon_{k}+E$ associated with the system having energy $\varepsilon_{k}$ and the bath being in the energy window $E$. We denote the corresponding probability of being in the energy shell $E_{\text {tot }}$ as $P\left(E_{\mathrm{tot}}\right):=\sum_{k} p\left(\varepsilon_{k}, E_{\mathrm{tot}}-\varepsilon_{k}\right)$. From Eq. (30), it follows that

$$
\partial_{t} P\left(E_{\mathrm{tot}}\right)=0
$$

and therefore the probability of being in the energy shell $E_{\text {tot }}$ is a conserved quantity of the evolution. In particular, its average $U:=\sum_{k, E} P\left(E_{\text {tot }}\right) E_{\text {tot }}$ fulfills $d U / d t=0$. Hence, the two variables $\varepsilon_{k}$ and $E$ are not independent and, given $E_{\text {tot }}$, one can obtain $\varepsilon_{k}$ from $E$ or vice versa. 


\section{B. Equilibrium states and local detailed balance}

From Eq. (30), the rate to jump from the state $\left(\varepsilon_{q}, E^{\prime}\right)$ to the state $\left(\varepsilon_{k}, E\right)$ is $W_{k q}\left(E, E^{\prime}\right) / V_{E^{\prime}}$. Using the symmetry property of the transition rates $W_{k q}\left(E, E^{\prime}\right)=W_{q k}\left(E^{\prime}, E\right)$, we find that the ratio of the rates to jump from the state $\left(\varepsilon_{k}, E\right)$ to the state $\left(\varepsilon_{q}, E+\omega_{k q}\right)$ reduces to

$$
\frac{V_{E}}{V_{E+\omega_{k q}}}=e^{-\left[\mathcal{S}_{\text {mic }}\left(E+\omega_{k q}\right)-\mathcal{S}_{\text {mic }}(E)\right]},
$$

where we have introduced the microcanonical (or Boltzmann) entropy $\mathcal{S}_{\text {mic }}(E)=\log V_{E}$. Equation (33) constitutes the local detailed balance condition for the EMME. Note that the local detailed balance condition in Eq. (33) matches the one obtained within the context of classical Markovian dynamics in phase space [30].

At equilibrium all probability flows are balanced and the local detailed balance condition implies

$$
\frac{p_{\mathrm{eq}}\left(\varepsilon_{k}, E\right)}{p_{\mathrm{eq}}\left(\varepsilon_{q}, E+\omega_{k q}\right)}=e^{-\left[\mathcal{S}_{\mathrm{mic}}\left(E+\omega_{k q}\right)-\mathcal{S}_{\mathrm{mic}}(E)\right]}
$$

for the equilibrium probabilities. Using the total energy $E_{\text {tot }}$ introduced above, we can write the steady-state condition in a more symmetric manner as

$$
\frac{p_{\text {eq }}\left(\varepsilon_{k}, E_{\mathrm{tot}}-\varepsilon_{k}\right)}{p_{\mathrm{eq}}\left(\varepsilon_{q}, E_{\mathrm{tot}}-\varepsilon_{q}\right)}=\frac{V_{E_{\mathrm{tot}}-\varepsilon_{k}}}{V_{E_{\mathrm{tot}}-\varepsilon_{q}}} .
$$

Since $P\left(E_{\text {tot }}\right)$ is constant, the final energy distribution for each of the probabilities $p\left(\varepsilon_{k}, E_{\text {tot }}\right)$ is fixed to have the equilibrium value

$$
p_{\mathrm{eq}}\left(\varepsilon_{k}, E_{\mathrm{tot}}-\varepsilon_{k}\right)=P\left(E_{\mathrm{tot}}\right) \frac{V_{E_{\mathrm{tot}}-\varepsilon_{k}}}{\sum_{q} V_{E_{\mathrm{tot}}-\varepsilon_{q}}},
$$

which was first noted in Ref. [16]. In summary, the steady-state condition in Eq. (35) implies that the system explores equiprobably all the available phase space given the macroscopic constraint that the total energy equals $E_{\text {tot }}$.

One may wonder whether the steady state of the EMME deviates from the steady state of the conventionally used BMS master equation. From Eq. (34), introducing the definition of the microcanonical (or Boltzmann) temperature $d E=T_{\text {mic }}(E) d \mathcal{S}_{\text {mic }}(E)$, we obtain for small $\omega_{k q}$

$$
\frac{p_{\mathrm{eq}}\left(\varepsilon_{k}, E\right)}{p_{\mathrm{eq}}\left(\varepsilon_{q}, E+\omega_{k q}\right)}=e^{-\omega_{k q} / T_{\mathrm{mic}}(E)} .
$$

This still involves the joint probability distributions of the system and the bath. If the bath energies are restricted to a microscopically large but macroscopically small energy range, we can assume that $T_{\text {mic }}$ depends only very slowly on $E$ such that it is a constant to first order. In that scenario, the population ratio in Eq. (37) is independent of the bath energy $E$. Assuming an unbounded bath spectrum, one can multiply Eq. (37) by $p\left(\varepsilon_{q}, E+\omega_{k q}\right)$ and then sum over $E$ to obtain

$$
\frac{p_{\mathrm{eq}}\left(\varepsilon_{k}\right)}{p_{\mathrm{eq}}\left(\varepsilon_{q}\right)}=e^{-\omega_{k q} / T_{\mathrm{mic}}}\left(1-\sum_{E=0}^{\omega_{k q}} \frac{p\left(\varepsilon_{q}, E\right)}{p\left(\varepsilon_{q}\right)}\right),
$$

where we have taken, without loss of generality, $\omega_{k q}>0$. The second term in large parentheses in Eq. (38) is a correction that appears due to the strict energy conservation condition $\partial_{t} P\left(E_{\mathrm{tot}}\right)=0$. Namely, for $E<\omega_{k q}$, the process $\varepsilon_{q} \mapsto \varepsilon_{k}$ cannot occur because there is no bath transition that can supply the energy deficit $\omega_{k q}$. In general, the contribution of the correction term will be small as long as the bath has initially a sufficiently high energy.

Instead, the conventional BMS master equation derived using the projector $\mathcal{P}_{\text {Born }}$ in Eq. (3) predicts the steady state

$$
\frac{p_{\mathrm{eq}}\left(\varepsilon_{k}\right)}{p_{\mathrm{eq}}\left(\varepsilon_{q}\right)}=e^{-\omega_{k q} / T_{\mathrm{can}}},
$$

where $T_{\text {can }}$ is fixed by the choice of the projector in Eq. (3). At first glance, the steady-state probabilities in Eqs. (38) and (39) are similar. Indeed, if the back action of the system on the bath is negligible and if the equivalence of ensembles holds, then $T_{\text {can }}=T_{\text {mic }}$, but we remark that $T_{\text {mic }} \neq T_{\text {can }}$ in general. This is best illustrated by the extreme case of negative-temperature $\left(T_{\text {mic }}<0\right)$ steady states, for which the energy populations increase with energy. Those negative-temperature states arise when, at least locally, the volume terms of the bath decrease with energy $V_{E}<V_{E^{\prime}}$ for $E>E^{\prime}$. Then the equivalence of ensembles clearly breaks down, as there is typically no Gibbs state that approximates the true state of the bath.

Following the observation of negative-temperature states [31], the question of whether negative temperatures are thermodynamically consistent has attracted much attention recently, with arguments presented against [3234] or in favor [35-37] of this. The debate remained, however, mostly on an abstract and axiomatic level. We here contribute to this fundamental question by numerically observing the emergence of stable population inverted steady states of an open system (see below). By "stable" we mean that all initial states of the open system tend to this population inverted state in the long-time limit (unless additional symmetries are present preventing the existence of a unique steady state). This result is also supported by the exact numerical integration of the full Schrödinger equation (see the second row in Fig. 2). These states match a Gibbs distribution with negative temperature if one uses the Boltzmann entropy to define temperature. In Sec. V we also formulate nonequilibrium first and second law for the EMME. Thus, within our framework we can 
deal with negative-temperature states without observing the emergence of any thermodynamic inconsistencies.

\section{System-bath correlations}

In the literature, the use of the uncorrelated projection superoperator $\mathcal{P}_{\text {Born }}$ is often justified by invoking the weak coupling assumption. However, as we show in Sec. V, this is not true, and even at the weak coupling limit strong system-bath correlations can build up (see also Ref. [38]). The EMME allows us to access part of these correlations and also to quantify them. We proceed as follows.

To quantify the (possibly quantum) system-bath correlations, we introduce the always positive quantum mutual information

$$
\mathcal{I}^{\mathrm{S}: \mathrm{B}}[\rho]:=\operatorname{tr}\left\{\rho\left[\log \rho-\log \left(\rho_{\mathrm{S}} \otimes \rho_{\mathrm{B}}\right)\right]\right\} \geq 0
$$

Then a high value of the mutual information indicates that the uncorrelated projector $\mathcal{P}_{\text {Born }}$ fails to capture the correlated nature of the system-bath dynamics, and therefore, the correctness of the BMS description is not guaranteed.

In absence of quantum correlations, the quantum mutual information is bounded from above by $\mathcal{I}^{\mathrm{S}: \mathrm{B}} \leq \log d_{\mathrm{S}}$, where $d_{\mathrm{S}}$ is the dimension of the system Hilbert space (naturally, we assume the dimension of the bath $d_{\mathrm{B}}$ is larger than the system dimension $d_{\mathrm{S}}$ ). Thus, in our context a value $\mathcal{I}^{\mathrm{S}: \mathrm{B}} \lesssim \log d_{\mathrm{S}}$ corresponds to strong system-bath correlations.

The EMME, however, keeps track of only part of the full system-bath dynamics and, ultimately, has information about the classical probability distribution $p\left(\varepsilon_{k}, E\right)$, with $p\left(\varepsilon_{k}, E\right)=\operatorname{tr}\left(\rho|k\rangle\langle k| \otimes \Pi_{E} / V_{E}\right)$. The system-bath correlations included in $p\left(\varepsilon_{k}, E\right)$ are quantified with use of the coarse-grained mutual information

$$
\mathcal{I}_{\mathrm{CG}}^{\mathrm{S}: \mathrm{B}}(\mathbf{p}):=\sum_{k, E} p\left(\varepsilon_{k}, E\right) \log \left(\frac{p\left(\varepsilon_{k}, E\right)}{p\left(\varepsilon_{k}\right) p(E)}\right) \geq 0
$$

where $\mathbf{p}$ is the vector with components $p\left(\varepsilon_{k}, E\right)$. It can be shown (see Appendix D) that

$$
\mathcal{I}^{\mathrm{S}: \mathrm{B}}[\rho] \geq \mathcal{I}_{\mathrm{CG}}^{\mathrm{S}: \mathrm{B}}(\mathbf{p}) \geq 0,
$$

where the first inequality becomes an equality for the state $\rho=\sum_{k, E} p\left(\varepsilon_{k}, E\right)|k\rangle\langle k| \otimes \Pi_{E} / V_{E}$.

Physically speaking, the reason why we can observe strong system-bath correlations with the EMME arises from the fact that the total energy $E_{\text {tot }}$ is conserved under the evolution, which constraints the values that the bath energy $E$ can take given a system energy $\varepsilon_{k}$. Hence, this constrained dynamics can give rise to high system-bath correlations as we numerically observe in Sec. V (see Fig. 5).

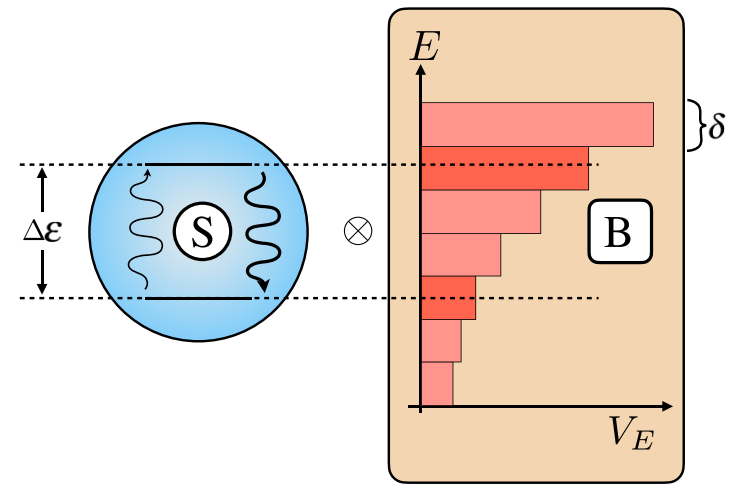

FIG. 1. Model of a spin coupled to a structured environment.

\section{EXAMPLE: SPIN COUPLED TO A STRUCTURED ENVIRONMENT}

To numerically check the validity of various results derived in Secs. III and V, we consider here an extension of the model studied in Ref. [6]. First, we derive the EMME for this model and then we compare its prediction with the exact integration of the Schrödinger equation. The model consists of a single spin with two energy levels $|0\rangle$ and |1) coupled to a finite environment (see Fig. 1). For convenience, we introduce the raising and lowering operators $\sigma_{+}=|1\rangle\langle 0|=\sigma_{-}^{\dagger}$. The bare system and bath Hamiltonians are

$$
\begin{aligned}
& \mathrm{H}_{\mathrm{S}}=\varepsilon_{0}|0\rangle\left\langle 0\left|+\varepsilon_{1}\right| 1\right\rangle\langle 1|, \\
& \mathrm{H}_{\mathrm{B}}=\sum_{E_{i}} E_{i}\left|E_{i}\right\rangle\left\langle E_{i}\right| .
\end{aligned}
$$

We consider the interaction $\mathrm{H}_{\text {int }}=V=\lambda\left(\sigma_{+}+\sigma_{-}\right) \otimes B$, and model the bath coupling operator using the RMT approach described in Sec. II C 2 in such a way that $\mathrm{B}$ is given by Eq. (26). This choice leads to $\delta \mathrm{H}(E)=0$ for all energies $E$. Recall that $c\left(E_{i}, E_{j}\right)$ are independent and identically distributed complex random numbers with zero mean and variance $a^{2}$. For simplicity, we assume that $\varepsilon_{1}-\varepsilon_{0}=\Delta \varepsilon=n \delta$, with $n \in \mathbb{N}$, which ensures the existence of an energy $E^{\prime}$ such that $E^{\prime}=E+\Delta \varepsilon$.

Remarkably, it is relatively simple to obtain the finitetime Redfield version of the EMME analytically for this system. As explained in Appendix B, this is achieved by conveniently introducing the function

$$
\zeta(t):=\frac{\delta}{\pi} \int_{0}^{t} \frac{\sin ^{2}(\delta \tau / 2)}{(\delta \tau / 2)^{2}} d \tau,
$$

which fulfills $\zeta(t \rightarrow \infty)=1$. Then the finite-time Redfield of the EMME takes the same form as Eq. (5) but the dissipation rates are multiplied by the time envelope $\zeta(t)$. For our particular example, this procedure leads to 


$$
\begin{aligned}
\partial_{t} \rho_{\mathrm{S}}(E)= & -i\left[\mathrm{H}_{\mathrm{S}}^{\prime}(E), \rho_{\mathrm{S}}(E)\right]+\zeta(t) \gamma(E, E+\Delta \varepsilon)\left(\frac{\sigma_{+} \rho_{\mathrm{S}}(E+\Delta \varepsilon) \sigma_{-}}{V_{E+\Delta \varepsilon}}-\frac{\left\{\rho_{\mathrm{S}}(E),|1\rangle\langle 1|\right\}}{2 V_{E}}\right) \\
& +\zeta(t) \gamma(E, E-\Delta \varepsilon)\left(\frac{\sigma_{-} \rho_{\mathrm{S}}(E-\Delta \varepsilon) \sigma_{+}}{V_{E-\Delta \varepsilon}}-\frac{\left\{\rho_{\mathrm{S}}(E),|0\rangle\langle 0|\right\}}{2 V_{E}}\right)
\end{aligned}
$$

where we have introduced $\gamma\left(E, E^{\prime}\right)=2 \pi \lambda^{2}\left(\left|b\left(E, E^{\prime}\right)\right|^{2}+\right.$ $\left.a^{2}\right) V_{E} V_{E^{\prime}} / \delta$. The function $\gamma\left(E, E^{\prime}\right)$ has the property $\gamma\left(E, E^{\prime}\right)=\gamma\left(E^{\prime}, E\right)$, and it vanishes if either $E$ or $E^{\prime}$ does not exist. Physically speaking, the second line of Eq. (45) represents a process in which a quantum of bath energy excites the system, while the third line represents the opposite process, in which the system gets de-excited. Equation (45) corresponds to the finite-time Redfield equation for this particular model, and we provide its analytical solution in Appendix E.

To benchmark the EMME, we proceed to investigate numerically a particular case of the model described above. Namely, we consider an environment of only two energy windows of macroscopic energies $E=\left\{\varepsilon_{0}, \varepsilon_{0}+\right.$ $\Delta \varepsilon$, with width $\delta=\Delta \varepsilon / 2$. The bath coupling operator B takes the form in Eq. (26), where we set for simplicity $b\left(E, E^{\prime}\right)=0$ and $a^{2}=1$. Also, we fix the initial state to $\rho(0)=|1\rangle\langle 1| \otimes \Pi_{\varepsilon_{0}} / V_{\varepsilon_{0}}$. For this particular choice, even if we were to consider more bands in the environment, their populations would not change with time. Finally, in the spirit of RMT, the numerical investigation is done with a single realization of the bath coupling operator $B$.

To challenge the assumptions made in the derivation, we consider the following three scenarios: (i) regular bath spectrum - the energy levels of the bath are equidistantly distributed (as in Ref. [6]); (ii) random bath spectrum - the energy levels of the bath are randomly distributed within each energy window; and (iii) half-filled energy window - not only are the bath energy levels randomly distributed but also the initial state has only half of the energy window occupied. Hence, we have $\mathcal{P}[\rho(0)] \neq \rho(0)$, but we still use the EMME from Eq. (5), ignoring any inhomogeneous contribution.

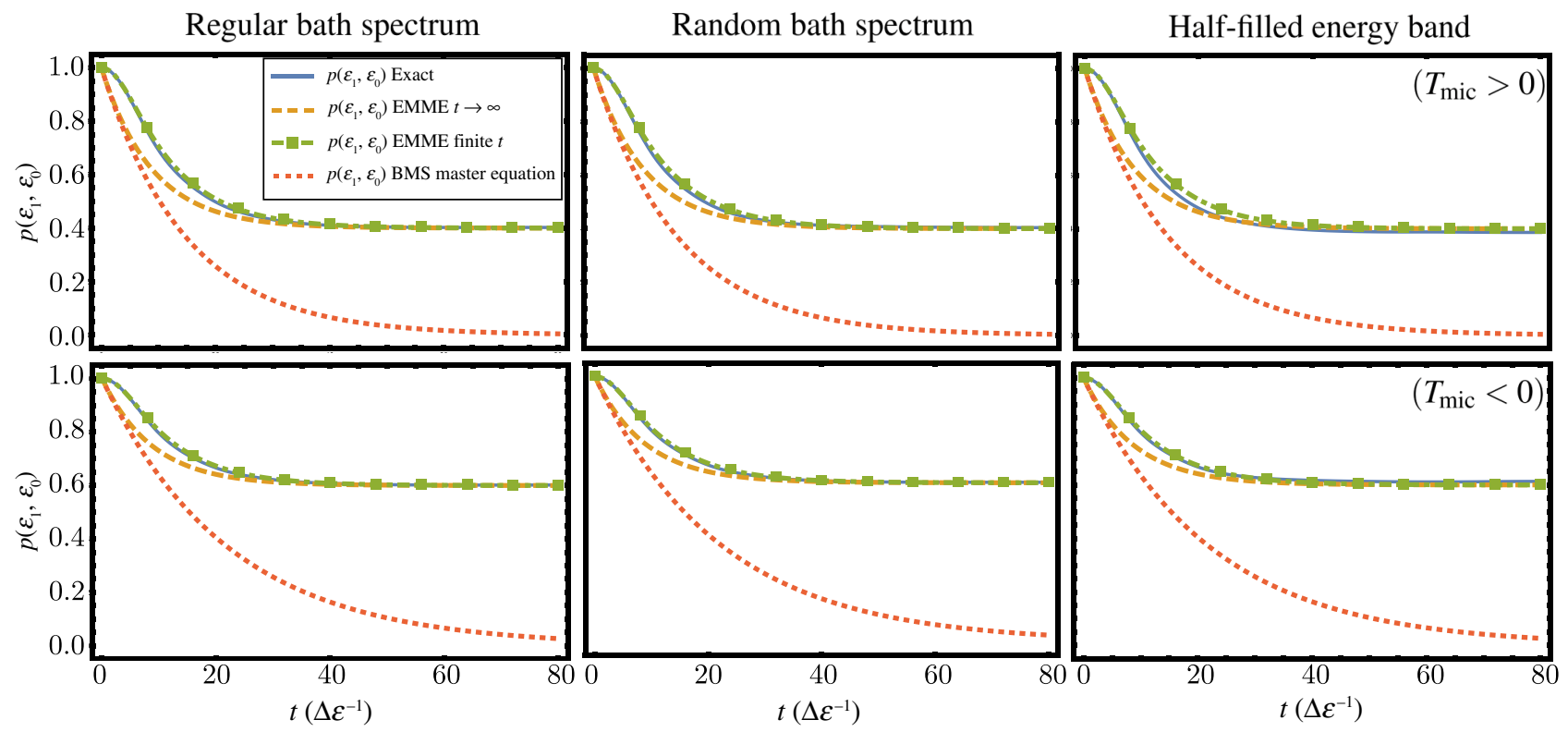

FIG. 2. Comparison of the evolution of the joint probability $p\left(\varepsilon_{1}, E=\varepsilon_{0}\right)$ for a spin system coupled to a two-band environment with initial state $\rho(0)=|1\rangle\langle 1| \otimes \Pi_{\varepsilon_{0}} / V_{\varepsilon_{0}}$, and $b\left(E, E^{\prime}\right)=0$ for all $E$ and $E^{\prime}$. The results are obtained with the following three different methods: exact evolution solving the Schrödinger equation (solid blue line), solution using the EMME with the Markov approximation $t \rightarrow \infty$ (dashed orange line), solution using the finite-time Redfield version of the EMME (dot-dashed green line with square markers), and solution using the standard BMS master equation (dotted red line). The first column corresponds to a regular bath with equidistant energy levels. The second column corresponds to randomly distributed energy levels. The third column corresponds to randomly distributed energy levels and the initial state of the bath taken to be only half-filled (i.e., a mixed stated of the $V_{\varepsilon_{0}} / 2$ levels with lower energy). The first row corresponds to $V_{\varepsilon_{0}}=400$ and $V_{\varepsilon_{1}}=600$. The second row corresponds to $V_{\varepsilon_{0}}=600$ and $V_{\varepsilon_{1}}=400$. $\lambda=3 \times 10^{-3}, \delta=0.5, \Delta \varepsilon=1$, and $a=1$. 
To be precise, the regular bath spectrum has energies $E_{i_{k}}=\varepsilon_{k}-\delta / 2+i_{k} \delta / V_{\varepsilon_{k}}$, where $i_{k} \in\left\{0,1, \ldots, V_{\varepsilon_{k}}-\right.$ 1 ) for $k=0,1$; the random bath spectrum has energies sampled from a flat distribution in the energy window $\left[\varepsilon_{k}-\delta / 2, \varepsilon_{k}+\delta / 2\right)$ for $k=0,1$; and the initial state of the half-filled energy window is taken to be $\rho(0)=|1\rangle\left\langle 1\left|\otimes \sum_{i=1}^{V \varepsilon_{0} / 2}\right| E_{i}\right\rangle\left\langle E_{i}\right|$. The situations described in (i)-(iii) increasingly challenge the assumptions made in the derivation of the EMME. We numerically compare them in Fig. 2 (one for each column).

In the first row in Fig. 2, we set $V_{\varepsilon_{0}}=400<V_{\varepsilon_{1}}=600$ and observe that the prediction of Eq. (45) (dot-dashed green line with square markers) agrees very well with the exact result (solid blue line) even for the random bath spectrum or the half-filled window. For comparison, we also plot the evolution of $p\left(\varepsilon_{1}, \varepsilon_{0}\right)$ as predicted by the more standard BMS master equation. To compute it, we use the projector in Eq. (3) where the state of the bath has been replaced by the microcanonical state $\Pi_{\varepsilon_{0}} / V_{\varepsilon_{0}}$, and we perform standard approximations (see Ref. [6] for details). As seen in Fig. (2), the standard BMS master equation (dotted red line) fails to capture the dynamics of the energy populations for this model. If one sets $\zeta(t) \mapsto 1$ (dashed orange line), as opposed to keeping the time-dependent dissipation rates $\zeta(t)$ (dot-dashed green line with square markers), the equation fails to describe the dynamics only at short timescales, but correctly predicts the steady state. This short-time behavior is ultimately a consequence of the failure of the Fermi golden rule and has a universal character [39].

Note that Ref. [6] corresponds to this reference with label Breuer 2006. In the second row in Fig. 2, we exchange the volumes of the bands such that $V_{\varepsilon_{0}}=600>$ $V_{\varepsilon_{1}}=400$. In all three scenarios, the EMME describes accurately the dynamics and predicts correctly the steady state. In this case, the equilibrium state shows population inversion and, in agreement with the discussion in Sec. III $\mathrm{B}$, it can be described by a negative-temperature state $T_{\text {mic }}<0$.

In Appendix F, we challenge the EMME even further by considering weaker and stronger coupling strengths $\lambda$, as well as smaller volumes $V_{E}$ for the energy windows of the bath. Even though the EMME is not able to always reproduce the dynamics accurately, for large volumes $V_{E} \gtrsim 100$, it does typically give the right timescale of decay and a good approximation for the steady-state populations.

\section{NONEQUILIBRIUM THERMODYNAMICS}

In Sec. II we derived the EMME starting from a microscopic description of the system and the bath. One motivation to derive such a master equation is its potential to describe small quantum devices, including heat engines, refrigerators, and heat pumps. If the bath is finite, operating those small quantum devices can cause the bath to develop nonequilibrium features during the evolution, and then the standard approach relying on a large bath in a Gibbs state cannot be applied. In this respect, it is important to obtain a consistent (nonequilibrium) thermodynamic interpretation of the dynamics.

Our master equation can describe three sources of nonequilibrium: (i) a nonthermal initial state $\rho(0)$ of the system and bath composite as considered in the numerical simulations above; (ii) a time-dependent system energy spectrum $\varepsilon_{k}\left(\lambda_{t}\right)$, where $\lambda_{t}$ represents a sufficiently slow driving protocol $\left(\dot{\lambda}_{t} \ll 1\right)$ such that one can directly replace $\varepsilon_{k} \mapsto \varepsilon_{k}\left(\lambda_{t}\right)$ in Eq. (5); and (iii) the system $\mathrm{S}$ being in contact with multiple environments (which we discuss in Sec. VI). We devote this part of the article to connect the nonequilibrium quantum dynamics of the EMME with the laws of thermodynamics.

\section{A. The first law of thermodynamics}

We start with the definition of the internal energy of the universe:

$$
U(t):=\sum_{k, E}\left[\varepsilon_{k}\left(\lambda_{t}\right)+E\right] p\left(\varepsilon_{k}, E\right) .
$$

By the first law of thermodynamics, its change can only be due to the mechanical work done on the system. Then, using $\partial_{t} P\left(E_{\text {tot }}\right)=0$, we obtain

$$
\frac{d}{d t} U=\dot{W}=\sum_{k, E}\left[\partial_{t} \varepsilon_{k}\left(\lambda_{t}\right)\right] p\left(\varepsilon_{k}, E\right) .
$$

We also introduce the internal energy of the system:

$$
U_{\mathrm{S}}(t):=\sum_{k, E} \varepsilon_{k}\left(\lambda_{t}\right) p\left(\varepsilon_{k}, E\right)
$$

Since the system $\mathrm{S}$ is in contact with a bath, the change in its internal energy is now due to work and heat:

$$
\frac{d}{d t} U_{\mathrm{S}}=\dot{W}+\dot{Q}
$$

Then the heat flux is found to be

$$
\dot{Q}:=-\sum_{k, E} E \partial_{t} p\left(\varepsilon_{k}, E\right)=\sum_{k, E} \varepsilon_{k}\left(\lambda_{t}\right) \partial_{t} p\left(\varepsilon_{k}, E\right),
$$

where the second equality follows again from $\partial_{t} P\left(E_{\text {tot }}\right)=$ 0 .

\section{B. The second law of thermodynamics}

The second law of thermodynamics states that a change in the thermodynamic entropy of the universe is always 
non-negative. However, there is no general consensus on the microscopic definition of the thermodynamic entropy. Here we use the recently (re)discovered observational entropy [40-43]:

$$
\mathcal{S}_{\text {obs }}(\mathbf{p}):=\sum_{k, E} p\left(\varepsilon_{k}, E\right)\left[-\log p\left(\varepsilon_{k}, E\right)+\log V_{E}\right],
$$

which also appears in the work of von Neumann and Wigner [12]. Here we use $\mathbf{p}$ to denote the vector of probabilities $p\left(\varepsilon_{k}, E\right)$ and we also write $\mathcal{S}_{\text {obs }}(t)$ instead of $\mathcal{S}_{\text {obs }}[\mathbf{p}(t)]$ when its meaning is clear from the context. We note that the observational entropy $\mathcal{S}_{\text {obs }}$ coincides with the well-known von Neumann entropy

$$
\mathcal{S}_{\mathrm{vN}}[\rho]:=-\operatorname{tr}(\rho \log \rho)
$$

when $\rho$ is diagonal and fulfills $\left\langle k, E_{i}|\rho| k, E_{i}\right\rangle=p\left(\varepsilon_{k}, E\right) / V_{E}$ for all $E_{i} \in E_{\delta}$. Finally, we introduce the coarse-grained relative entropy

$$
\mathcal{D}_{\mathrm{CG}}(\mathbf{p} \| \mathbf{q}):=\sum_{k, E} p\left(\varepsilon_{k}, E\right) \log \frac{p\left(\varepsilon_{k}, E\right)}{q\left(\varepsilon_{k}, E\right)} \geq 0
$$

for two arbitrary vectors of probabilities $\mathbf{p}$ and $\mathbf{q}$.

Equipped with those information-theoretical quantities, our aim is to derive a second law in terms of the observational entropy $\mathcal{S}_{\text {obs }}$, and hence we define the entropy production rate

$$
\dot{\Sigma}:=\frac{d}{d t} \mathcal{S}_{\text {obs }}(t)
$$

In contrast to Ref. [43], where only the integrated change $\Delta \mathcal{S}_{\text {obs }}(t)=\mathcal{S}_{\text {obs }}(t)-\mathcal{S}_{\text {obs }}(0)$ in observational entropy was shown to be positive, we derive here the stronger result that the entropy production rate is always positive $\dot{\Sigma} \geq 0$. To prove the positivity of $\dot{\Sigma}$, it is convenient to introduce the joint Gibbs distribution at temperature $T$ :

$$
p_{T}\left(\varepsilon_{k}\left(\lambda_{t}\right), E\right):=\frac{V_{E} \exp \left\{-\left[\varepsilon_{k}\left(\lambda_{t}\right)+E\right] / T\right\}}{Z_{\mathrm{S}}\left(\lambda_{t}\right) Z_{\mathrm{B}}},
$$

where $Z_{\mathrm{S}}\left(\lambda_{t}\right):=\sum_{k} \exp \left[-\varepsilon_{k}\left(\lambda_{t}\right) / T\right]$ and $Z_{\mathrm{B}}:=\sum_{E} V_{E}$ $\exp (-E / T)$ are the partition functions. Note that $\mathbf{p}_{T}\left(\lambda_{t}\right)$ is a stationary distribution since it fulfills the steady-state condition in Eq. (35). Below, we show that it is possible to recast the entropy production rate as

$$
\dot{\Sigma}=-\left.\partial_{t}\right|_{\lambda_{t}} \mathcal{D}_{\mathrm{CG}}\left[\mathbf{p}(t)|| \mathbf{p}_{T}\left(\lambda_{t}\right)\right]
$$

where the symbol $\left.\partial_{x}\right|_{y}$ stands for the partial derivative with respect to $x$ while $y$ is kept fixed. The result above then implies that the entropy production rate is non-negative:

$$
\frac{d}{d t} \mathcal{S}_{\text {obs }}(t)=\dot{\Sigma} \geq 0
$$

This follows from two facts: first, $\mathbf{p}_{T}$ is an equilibrium state of the dynamics and, second, the dynamics are Markovian, which implies that we can use monotonicity of the relative entropy. If either of these assumptions is violated, negative entropy production rates can appear although $\Sigma(t)=$ $\Delta \mathcal{S}_{\text {obs }}(t)$ remains positive [44].

The proof is as follows. We start by writing the classical relative entropy in Eq. (56) in terms of the observational entropy as

$$
\begin{aligned}
\mathcal{D}_{\mathrm{CG}}\left[\mathbf{p}(t) \| \mathbf{p}_{T}\left(\lambda_{t}\right)\right]= & -\mathcal{S}_{\text {obs }}(t)+T^{-1} U(t) \\
& +\log Z_{\mathrm{S}}\left(\lambda_{t}\right)+\log Z_{\mathrm{B}} .
\end{aligned}
$$

Then, using the chain rule $d / d t=\partial_{t}+\dot{\lambda}_{t} \partial_{\lambda_{t}}$, we obtain

$$
\left.\partial_{t}\right|_{\lambda_{t}} \mathcal{D}_{\mathrm{CG}}\left[\mathbf{p}(t)|| \mathbf{p}_{T}\left(\lambda_{t}\right)\right]=\frac{d}{d t} \mathcal{S}_{\mathrm{obs}}(t)-\frac{1}{T}\left(\frac{d U}{d t}-\dot{W}\right) .
$$

Finally, we note that the rightmost term in Eq. (59) vanishes on the use of the first law in Eq. (47). Hence, we obtain the second law of thermodynamics in Eq. (57), which implies a positive entropy production rate. The second law $\dot{\Sigma}(t) \geq 0$ derived here holds as long as $\mathcal{P}[\rho(0)]=$ $\rho(0)$ and, in particular, we do not invoke at any time the assumption of thermal equilibrium for the bath.

\section{Connecting the first and second law}

In the above subsections, we derived independently the first and second law of thermodynamics and, at this point, they appear rather disconnected. In standard phenomenological thermodynamics, the first and second law are related through the well-known Clausius inequality. Namely, if during a transformation the bath is well approximated at all times $t$ by an equilibrium state at temperature $T_{\mathrm{B}}(t)$, then the Clausius inequality reads

$$
\Delta \mathcal{S}^{\mathrm{S}}(t)-\int_{0}^{t} d t^{\prime} \frac{\dot{Q}\left(t^{\prime}\right)}{T_{\mathrm{B}}\left(t^{\prime}\right)} \geq 0
$$

where $\mathcal{S}^{S}$ denotes the system thermodynamic entropy. Equation (60) connects the entropic changes with the heat flux into the system $\dot{Q}$ when the environment is at temperature $T_{\mathrm{B}}$. In particular, a large environment with an infinite heat capacity would keep its temperature constant throughout the process [i.e., $\left.T_{\mathrm{B}}(t) \approx T_{\mathrm{B}}(0)\right]$. Then the Clausius 
inequality simplifies to

$$
\Delta \mathcal{S}^{\mathrm{S}}(t)-\frac{Q(t)}{T_{\mathrm{B}}(0)} \geq 0
$$

where $Q(t)=\int_{0}^{t} d t^{\prime} \dot{Q}$. Equation (61) is conventionally considered the second law in quantum thermodynamics. Importantly, Eq. (61) should be regarded as a consequence of the second law only under the conditions set out above.

In our description, during a transformation $\rho(0) \mapsto$ $\rho(t)$, the environment generically goes through several nonequilibrium states for which a temperature $T_{\mathrm{B}}$ may not even be defined. To establish a connection with the Clausius inequality, we define an effective nonequilibrium temperature $T_{\mathrm{B}}^{*}(t)$ by demanding that the actual bath energy matches the one of a fictitious canonical ensemble at that temperature. In the equations, $T_{\mathrm{B}}^{*}(t)$ is determined by our solving

$$
\sum_{E} E \frac{V_{E} e^{-E / T_{\mathrm{B}}^{*}(t)}}{Z_{\mathrm{B}}(t)}=\sum_{k, E} E p\left(\varepsilon_{k}, E ; t\right)=: U_{\mathrm{B}}(t) .
$$

Operationally, $T_{\mathrm{B}}^{*}(t)$ corresponds to the temperature of a superbath that if weakly coupled to the bath $\mathrm{B}$ would give rise to a total vanishing heat current between the bath and the superbath.

As emphasized above, the Clausius inequality follows from the second law, Eq. (57), only under additional approximations. To derive it rigorously, we assume the following: (i) $\rho(0)$ is a product state of the system and the bath, that is, $\rho(0)=\rho_{\mathrm{S}}(0) \otimes \rho_{\mathrm{B}}(0)$, and (ii) the initial bath state $\rho_{\mathrm{B}}(0)$ is a Gibbs state of the bath at temperature $T(0)$.

We start by noting that for a Gibbs state at an arbitrary temperature $T$, the following differential relation holds:

$$
d U_{\mathrm{B}}=T d \mathcal{S}_{\mathrm{obs}}^{\mathrm{B}}\left(\mathbf{p}_{T}\right) .
$$

Here we use the superscript B to indicate that $\mathcal{S}_{\text {obs }}^{\mathrm{B}}$ corresponds to the observational entropy of the bath alone. Next we note the following identity:

$$
\begin{aligned}
\Delta \mathcal{S}_{\mathrm{obs}}^{\mathrm{B}}(t)= & \mathcal{S}_{\mathrm{obs}}^{\mathrm{B}}(t)-\mathcal{S}_{\mathrm{obs}}^{\mathrm{B}}\left(\mathbf{p}_{T_{\mathrm{B}}^{*}(t)}\right) \\
& +\mathcal{S}_{\mathrm{obs}}^{\mathrm{B}}\left(\mathbf{p}_{T_{\mathrm{B}}^{*}(t)}\right)-\mathcal{S}_{\mathrm{obs}}^{\mathrm{B}}(0) .
\end{aligned}
$$

The difference of the first two terms of the above equation is negative since, by construction, $\mathbf{p}(t)$ and $\mathbf{p}_{T_{\mathrm{B}}^{*}(t)}$ have the same energy and the Gibbs state maximizes the entropy. Using the differential relation in Eq. (63), we can cast the last two terms of Eq. (64) as

$$
\mathcal{S}_{\mathrm{obs}}^{\mathrm{B}}\left(\mathbf{p}_{T_{\mathrm{B}}^{*}(t)}\right)-\mathcal{S}_{\mathrm{obs}}^{\mathrm{B}}\left(\mathbf{p}_{T_{\mathrm{B}}(0)}\right)=-\int_{0}^{t} d t^{\prime} \frac{\dot{Q}\left(t^{\prime}\right)}{T_{\mathrm{B}}^{*}\left(t^{\prime}\right)} .
$$

Moreover, using the coarse-grained mutual information, Eq. (41), as well as the initial product state assumption, we obtain the following relation:

$$
\Delta \mathcal{S}_{\mathrm{obs}}^{\mathrm{S}}(t)+\Delta \mathcal{S}_{\mathrm{obs}}^{\mathrm{B}}(t)=\Delta \mathcal{S}_{\mathrm{obs}}(t)+\mathcal{I}_{\mathrm{CG}}^{\mathrm{S}: \mathrm{B}}(\mathbf{p}(t)) \geq 0 .
$$

Finally, putting together Eqs. (64)-(66), we find the following chain of inequalities:

$$
\begin{aligned}
\Delta \mathcal{S}_{\mathrm{obs}}^{\mathrm{S}}(t)-\int_{0}^{t} d t^{\prime} \frac{\dot{Q}\left(t^{\prime}\right)}{T_{\mathrm{B}}^{*}\left(t^{\prime}\right)} & \geq \Delta \mathcal{S}_{\mathrm{obs}}^{\mathrm{S}}(t)+\Delta \mathcal{S}_{\mathrm{obs}}^{\mathrm{B}}(t) \\
& \geq \Delta \mathcal{S}_{\mathrm{obs}}(t) \geq 0
\end{aligned}
$$

which proves the aforementioned Clausius inequality and connects the first and second law. The first inequality in Eq. (67) becomes an exact equality whenever the bath does not develop any noticeable nonequilibrium features.

\section{Testing the results numerically}

To conclude this section, we test numerically the results derived above. We consider again the same spin system described in Sec. IV but now we allow the energies $\varepsilon_{k}\left(\lambda_{t}\right)$ to depend parametrically on time. For concreteness, we leave the energy $\varepsilon_{0}=0$ constant and quench periodically the energy of the excited state as

$$
\varepsilon_{1}\left(\lambda_{t}\right)=\left\{\begin{array}{cc}
\Delta \varepsilon & t \in\left[0, t_{\star}\right), \\
2 \Delta \varepsilon & t \in\left[t_{\star}, 2 t_{\star}\right),
\end{array}\right.
$$

where $2 t_{\star}$ is the period and $\Delta \varepsilon$ us a fixed energy splitting. Since we are quenching back and forth the energy of the excited state, three energy windows of the environment are now explored. We consider their associated macroscopic energies to be $E \in\{0, \Delta \varepsilon, 2 \Delta \varepsilon\}$.

In Fig. 3, we compare the exact dynamics with the prediction of the EMME. The volume terms of the bands are set to $V_{E} \in\{100,200,400\}$ and we chose the initial state $\rho(0)=|1\rangle\langle 1| \otimes \Pi_{E=0} / V_{E=0}$. We observe that the EMME is also able to reproduce accurately the dynamics when the system energy levels are periodically quenched. This justifies retrospectively our claim above that we can replace the static system energies $\varepsilon_{k}$ with time-dependent energies $\varepsilon_{k}\left(\lambda_{t}\right)$ as long as $\lambda_{t}$ varies slowly compared with the relaxation time of the bath.

During the evolution the system and bath develop nonequilibrium features, but the Clausius inequality and our second law in Eq. (57) remain valid at all times as demonstrated in Fig. 4. In the inset, we show the corresponding evolution of the non-equilibrium temperature of the bath $T_{\mathrm{B}}^{*}(t)$, which, due to the finite heat capacity of the bath, cannot be approximated by a constant value. The fact that we start with a zero effective temperature $T_{\mathrm{B}}^{*}(0)=0$ is a result of our choice for the initial state $\rho(0)$ and a consequence of the bath model, where we ignore any energy levels below the lowest band that participates in the dynamics. 


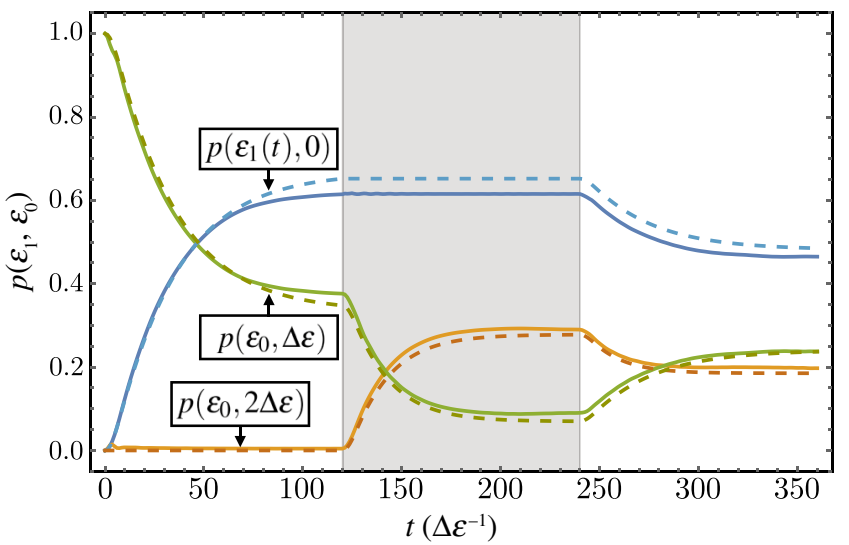

FIG. 3. Comparison of the exact dynamics (solid lines) with the dynamics predicted by the EMME (dashed lines) for a spin with energies $\varepsilon_{0}=0$ and $\varepsilon_{1}\left(\lambda_{t}\right)=\Delta \varepsilon$ (white background) and $\varepsilon_{1}\left(\lambda_{t}\right)=2 \Delta \varepsilon$ (shadowed background). See the main text for details. $\delta=0.5, t_{\star}=120 \Delta \varepsilon^{-1}, b\left(E, E^{\prime}\right)=0$ for all $E, E^{\prime}$, and $a=1$.

The difference between the dash-dotted green curve and the dashed orange curve in Fig. 4 is a nonequilibrium effect resulting from a bath state deviating from an ideal thermal state. The difference between the solid blue curve and the dashed orange curve in Fig. 4 is, instead, a result of the (ignored) classical system-bath correlations as measured by the coarse-grained mutual information in Eq. (41). To investigate the latter, we numerically compute the evolution of the mutual information. In Fig. 5, we show that $\mathcal{I}^{\mathrm{S}: \mathrm{B}}$ always upper bounds $\mathcal{I}_{\mathrm{CG}}^{\mathrm{S}}$, which increases close to their

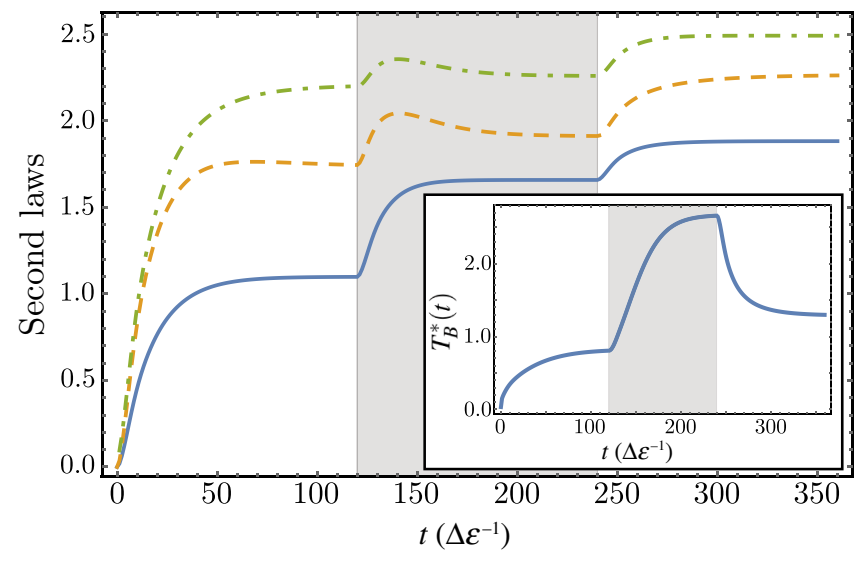

FIG. 4. Numerical proof of the Clausius inequality: $\Delta \mathcal{S}_{\text {obs }}(t)$ (solid blue line), $\Delta \mathcal{S}_{\mathrm{obs}}^{\mathrm{S}}(t)+\Delta \mathcal{S}_{\mathrm{obs}}^{\mathrm{B}}(t)$ (dashed orange line), and $\Delta \mathcal{S}_{\text {obs }}^{\mathrm{S}}(t)-\int_{0}^{t} d t^{\prime} \dot{Q}\left(t^{\prime}\right) / T_{\mathrm{B}}^{*}\left(t^{\prime}\right)$ (dot-dashed green line) computed using the EMME for a spin with energies $\varepsilon_{0}=0$ and $\varepsilon_{1}(t)=\Delta \varepsilon$ (white background) and $\varepsilon_{1}(t)=2 \Delta \varepsilon$ (shadowed background). See the main text for details. The inset shows the corresponding nonequilbrium temperature of the bath $T_{\mathrm{B}}^{*}(t)\left(k_{\mathrm{B}}=1\right)$ for the same protocol. $\delta=0.5, t_{\star}=120 \Delta \varepsilon^{-1}, b\left(E, E^{\prime}\right)=0$ for all $E, E^{\prime}$, and $a=1$.

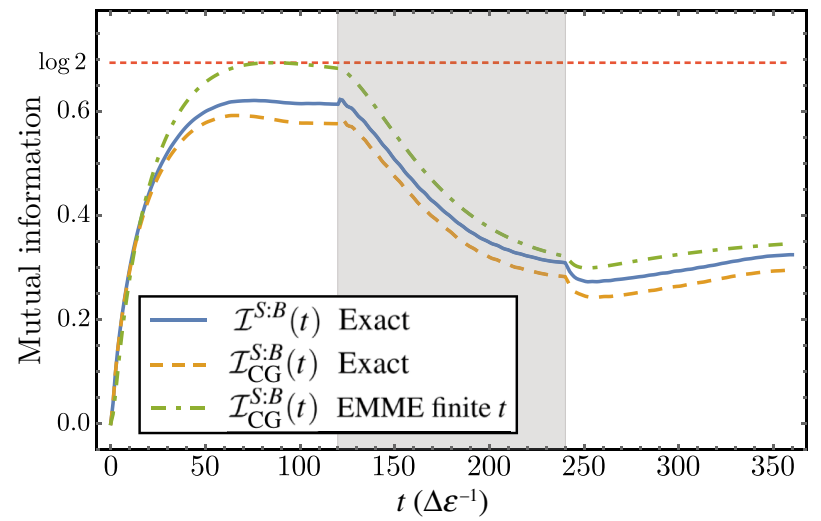

FIG. 5. Evolution of the mutual information comparing the exact solution of the quantum mutual information (solid blue line) with the exact coarse-grained mutual information (dashed orange line) and the approximation obtained using the EMME (dot-dashed green lines) for a spin with energies $\varepsilon_{0}=0$ and $\varepsilon_{1}\left(\lambda_{t}\right)=\Delta \varepsilon$ (white background) and $\varepsilon_{1}\left(\lambda_{t}\right)=2 \Delta \varepsilon$ (shadowed background). See the main text for details. The initial state is chosen as $\rho(0)=|1\rangle\langle 1| \otimes \Pi_{E=0} / V_{E=0} . \delta=0.5, t_{\star}=120 \Delta \varepsilon^{-1}$, $b\left(E, E^{\prime}\right)=0$ for all $E, E^{\prime}$, and $a=1$.

maximum value $\log 2$. Since the mutual information can grow close to its maximum value, the system-bath correlations are not negligible, showing that the weak-coupling approximation does not justify the use of an uncorrelated reference state of the system and the bath. Also, the EMME provides a good approximation of the real value of $\mathcal{I}_{\mathrm{CG}}^{\mathrm{S}: \mathrm{B}}$, but its prediction can violate the bound in Eq. (42). The reason that the EMME overestimates the system-bath correlation is a consequence of the strict energy conservation derived in Sec. III A, which is never exactly satisfied for any finite coarse graining and any finite system-bath coupling strength.

\section{GENERALIZATION TO MULTIPLE ENVIRONMENTS}

In the previous sections, we studied the case of a quantum system S in contact with a single heat bath B. Similar findings hold for the case of multiple environments and, for completeness, we outline in this section the generalization of the main results to multiple environments. Its detailed study including the treatment of various interesting applications is left for future work.

We consider multiple baths labeled by $v=1, \ldots, n$, with Hamiltonian $\mathrm{H}_{\mathrm{B}_{v}}$ coupled to the system with an interaction $\mathrm{H}_{\mathrm{int}, v}=\lambda S_{\nu} \otimes B_{\mathrm{int}, v}$ (again, more general expressions can be found in the Appendixes). Denoting $E_{v, i}$ as the eigenenergies of the $v$ th bath Hamiltonian, we proceed to coarse-grain the energies into energy windows $E_{v, \delta_{v}}=\left[E_{v}-\delta_{v} / 2, E_{v}+\delta_{v} / 2\right)$ centered at the energy $E_{v}$. 
Then we can define a projection operator

$$
\mathcal{P}[\rho]:=\sum_{\mathbf{E}} \rho_{\mathrm{S}}(\mathbf{E}) \otimes \frac{\Pi_{\mathbf{E}}}{V_{\mathbf{E}}}
$$

where the vector $\mathbf{E}=\left(E_{1}, \ldots, E_{n}\right)$, the projector $\Pi_{\mathbf{E}}=$ $\Pi_{E_{1}} \otimes \cdots \otimes \Pi_{E_{n}}$, and the joint volume $V_{\mathbf{E}}=V_{E_{1}} \cdots V_{E_{n}}$ have been introduced. All the steps followed in Sec. II are valid under the replacement $E \mapsto \mathbf{E}$ with the important remark that we obtain an additive structure for the EMME; that is, there are no crossed terms with $v \neq v^{\prime}$ (see Appendix G). For the multiple-bath scenario, the bath correlation function is given by

$$
C_{\mathrm{B}_{v}}\left(\mathbf{E}, \mathbf{E}^{\prime} ;-\tau\right):=\lambda^{2}\left\langle\tilde{B}_{v}^{\dagger}(-\tau) \Pi_{\mathbf{E}} B_{v}\right\rangle_{\mathbf{E}^{\prime}} .
$$

Note that $C_{\mathrm{B}_{v}}\left(\mathbf{E}, \mathbf{E}^{\prime} ;-\tau\right)$ is very sparse, since the vectors $\mathbf{E}$ and $\mathbf{E}^{\prime}$ can be different only in the $v$ th component. Therefore, it is possible to redefine complex decay rates $\gamma_{\nu}\left(E_{v}, E_{v}^{\prime} ; \omega\right)$ that depend only on the $v$ th component of the energy vectors $\mathbf{E}$ and $\mathbf{E}^{\prime}$. For the same reasons that we give in Sec. II, it is justified to factor $\gamma_{\nu}\left(E_{\nu}, E_{\nu}^{\prime} ; \omega\right)=$ $\gamma_{v}\left(E_{v}, E_{v}^{\prime}\right) \delta_{E_{v}^{\prime}, E_{v}+\omega}$, yielding the EMME

$$
\begin{aligned}
\partial_{t} \rho_{\mathrm{S}}(\mathbf{E}) \\
=-i\left[\mathrm{H}_{\mathrm{S}}^{\prime}(\mathbf{E}), \rho_{\mathrm{S}}(\mathbf{E})\right] \\
\quad+\sum_{\nu} \sum_{\omega}\left(\frac{\gamma_{v}\left(E_{v}, E_{v}-\omega\right)}{V_{E_{v}-\omega}} S_{v, \omega} \rho_{\mathrm{S}}\left(\mathbf{E}+\omega \hat{\mathbf{e}}_{v}\right) S_{v, \omega}^{\dagger}\right. \\
\left.\quad-\frac{\gamma_{v}\left(E_{v}+\omega, E_{v}\right)}{2 V_{E_{v}}}\left\{\rho_{\mathrm{S}}(\mathbf{E}), S_{v, \omega}^{\dagger} S_{v, \omega}\right\}\right)
\end{aligned}
$$

where $\hat{\mathbf{e}}_{v}$ is the unit vector along the $v$ th component.

From Eq. (71), it is possible to obtain the conservation of the probability $\partial_{t} P\left(E_{\text {tot }}\right)=0$, with $P\left(E_{\text {tot }}\right)=$ $\sum_{k, \mathbf{E}} p\left(\varepsilon_{k}, \mathbf{E}\right) \delta_{E_{\mathrm{tot}}, \varepsilon_{k}+\sum_{v} E_{v}}$, as well as a steady-state condition similar to Eq. (35) (further details are given in Appendix G).

The thermodynamic behavior studied in Sec. V also extends to multiple environments. Introducing $\dot{Q}_{\nu}=$ $\sum_{k, \mathbf{E}} E_{v} \partial_{t} p\left(\varepsilon_{k}, \mathbf{E}\right)$, we find the first law takes the form

$$
\frac{d}{d t} U_{\mathrm{S}}=\sum_{v} \dot{Q}_{v}+\dot{W} .
$$

To obtain the second law, we first need to introduce the multiple-bath observational entropy

$$
\mathcal{S}_{\text {obs }}(\mathbf{p})=\sum_{k, \mathbf{E}} p\left(\varepsilon_{k}, \mathbf{E}\right)\left[-\log p\left(\varepsilon_{k}, \mathbf{E}\right)+\log V_{\mathbf{E}}\right],
$$

for which it is possible to show that $d \mathcal{S}_{\text {obs }}(t) / d t \geq 0$. The proof uses the additive structure of the EMME, and then proceeds analogously to the single-bath case. Finally, the Clausius inequality is similarly found as

$$
\Delta \mathcal{S}_{\mathrm{obs}}^{\mathrm{S}}(t)-\sum_{\nu} \int_{0}^{t} d t^{\prime} \frac{\dot{Q}_{v}\left(t^{\prime}\right)}{T_{\nu}^{*}\left(t^{\prime}\right)} \geq \Delta \mathcal{S}_{\mathrm{obs}}(t) \geq 0
$$

\section{OUTLOOK AND COMPARISON WITH OTHER MASTER EQUATIONS}

We conclude by comparing the EMME with other master equation approaches. In the previous sections we discussed the EMME in comparison with the popular Redfield and BMS (or "quantum optical") master equations [1-3], whose dynamic and thermodynamic predictions can differ significantly from those of the EMME. Note that, similar in spirit to the EMME, the BMS master equation is sometimes refined by equipping it with an additional counting field, which keeps track of the changes in bath energy $[2,45]$. However, these counting field master equations make no further use of this information to obtain a more accurate system dynamics: after integrating out of the counting field, the reduced dynamics of the system is still given by the standard BMS master equation. Therefore, we focus her only on a comparison with more advanced master equations going beyond this standard approach.

Clearly, one way to obtain improved results is to use the standard projection operators $\mathcal{P}_{\text {Born }}$ as for the BMS master equation but to go beyond second order in the interaction Hamiltonian. However, this quickly becomes cumbersome and, as shown in Ref. [6], even the fourthorder master equation does not necessarily increase the accuracy, still giving qualitatively wrong results in comparison with the EMME and the exact solution. A more sophisticated idea in comparison to simply "crank up" the perturbative hierarchy is to apply different approximation techniques to the memory kernel in the Nakajima-Zwanzig equation based on, for example, semiclassical simulations of the bath dynamics $[46,47]$. While being nonperturbative and more accurate, this approximation so far mostly been used for numerical case-by-case studies and it seems hard to obtain general insights from it.

Another approach, which still resides in the standard picture by tracing out the entire bath and keeping only information about the system, is to combine second-order master equations and polaron transformations [48]. In this approach one first maps a strongly coupled systembath Hamiltonian to a weakly coupled one by using the polaron transformation and afterwards combines it with standard perturbative master equations. This allows one to treat strong coupling, as demonstrated, for example, in Refs. [49-52], but it does not overcome the Markov approximation and essentially treats the bath as being in (conditional) equilibrium throughout. Nevertheless, combining polaron transformations, which work well only for particular system-bath models, with the EMME seems to 
be a promising avenue for future research to treat strongly coupled systems in a more accurate way.

Finally, another master equation approach, which shares some similarities with our approach by explicitly treating parts of the bath degrees of freedom, is based on Markovian embedding strategies [53-55]. By redefining the system-bath partition and applying a master equation to an enlarged but weakly coupled and Markovian system, as done using, for example, the reaction coordinate master equation [56-60] or other formally exact but more involved master equations [61,62], one can obtain numerically accurate results while retaining at the same time detailed information about system-bath correlations and (parts of) the bath degrees of freedom. We have not yet benchmarked our master equation with these techniques, but we expect the latter to be more accurate. On the downside, these Markovian embedding strategies, as well as the aforementioned master equations using polaron transformations or semiclassical simulations of the memory kernel, all rely on the paradigmatic Caldeira-Leggett model. We believe it is a significant advantage that the EMME applies in principle to every system-bath model.

Thus, to summarize, the EMME opens up the possibility of treating a variety of interesting nonequilibrium situations, including, for example, finite heat baths, spin environments, nonlinear system-bath interactions, and impurities in quantum many body systems, in a dynamically more accurate way beyond the restrictive static bath and Markov approximation and with an intuitive and consistent thermodynamic interpretation. Perhaps in combination with other techniques, such as the ones just mentioned, we are convinced that it provides an efficient, flexible, and intuitive tool for future research in quantum nanotechnologies.

\section{ACKNOWLEDGMENTS}

We thank Massimiliano Esposito and Kavan Modi for stimulating discussions on this and related topics. We acknowledge financial support from the Spanish Agencia Estatal de Investigación, project PID2019-107609GBI00, Spanish MINECO FIS2016-80681-P (AEI/FEDER, UE), Generalitat de Catalunya CIRIT 2017-SGR-1127, and from Secretaria d'Universitats i Recerca del Departament d'Empresa i Coneixement de la Generalitat de Catalunya, co-funded by the European Union Regional Development Fund within the ERDF Operational Program of Catalunya (project QuantumCat, ref. 001-P-001644). ARC also acknowledges support from AGAUR-FI grant number FI-2018-B01134. PS also acknowledges support from the German Research Foundation (DFG) (project STR 1505/2-1).

\section{APPENDIX}

For the sake of explanation, we consider a single system-bath coupling operator throughout the main text.
For completeness, we set out here the most general form of the EMME for multiple environments and multiple coupling operators, corresponding to the interaction $\mathrm{H}_{\mathrm{int}, v}=$ $\lambda \sum_{\alpha_{v}} S_{v}^{\alpha_{v}} \otimes B_{\text {int }}^{\alpha_{v}}$. In this case, the EMME yields

$$
\begin{aligned}
& \partial_{t} \rho_{\mathrm{S}}(\mathbf{E})=-i\left[\mathrm{H}_{\mathrm{S}}^{\prime}(\mathbf{E}), \rho_{\mathrm{S}}(\mathbf{E})\right] \\
& +\sum_{\nu} \sum_{\alpha_{\nu} \alpha_{v}^{\prime}} \sum_{\omega}\left(\frac{\gamma_{\nu}^{\alpha_{\nu} \alpha_{v}^{\prime}}\left(E_{v}, E_{v}-\omega\right)}{V_{E_{v}-\omega}} S_{v, \omega}^{\alpha_{v}} \rho_{\mathrm{S}}\left(\mathbf{E}+\omega \hat{\mathbf{e}}_{v}\right) S_{v, \omega}^{\alpha_{v}^{\prime} \dagger}\right. \\
& \left.-\frac{\gamma_{\nu}^{\alpha_{\nu} \alpha_{v}^{\prime}}\left(E_{v}+\omega, E_{v}\right)}{2 V_{E_{v}}}\left\{\rho_{\mathrm{S}}(\mathbf{E}), S_{v, \omega}^{\alpha_{v}^{\prime} \dagger} S_{v, \omega}^{\alpha_{\nu}}\right\}\right)
\end{aligned}
$$

Equation (1) reduces to Eq. (71) for $\mathrm{H}_{\text {int, }, v}=\lambda S_{v} \otimes B_{\text {intv }}$ (for all $v$ ) and to Eq. (5) for a single bath index $v$.

\section{APPENDIX A: DETAILS ON THE DERIVATION OF THE EMME}

Here we provide details on the derivation of the EMME, the computation of the dissipation rates in the three approaches listed in the main text, and a comparison between them. For generality, we consider the interaction $\mathrm{H}_{\text {int }}=\lambda \sum_{\alpha} S^{\alpha} \otimes B_{\text {int }}^{\alpha}$, which appears as a double index $\alpha \alpha^{\prime}$ in the expression for the correlation functions [i.e., $\left.C_{\mathrm{B}}^{\alpha \alpha^{\prime}}\left(E, E^{\prime} ; \omega\right)\right]$ and the corresponding dissipation rates [i.e., $\left.\gamma^{\alpha \alpha^{\prime}}\left(E, E^{\prime}\right)\right]$. In the three methods, the aim is to compute the complex dissipation rates $\gamma^{\alpha \alpha^{\prime}}\left(E, E^{\prime}\right)$ starting from the correlation function

$$
C_{\mathrm{B}}^{\alpha \alpha^{\prime}}\left(E, E^{\prime} ;-\tau\right)=\sum_{E_{i} \in E_{\delta}} \sum_{E_{j} \in E_{\delta}^{\prime}} \frac{\lambda^{2}}{V_{E^{\prime}}} B_{i j}^{\alpha^{\prime} *} B_{i j}^{\alpha} e^{i\left(E_{i}-E_{j}\right) \tau} .
$$

\section{Details of the heuristic approach}

As explained in the main text, this approach is based on the substitution $E_{i}-E_{j} \mapsto E-E^{\prime}$ in Eq. (A1). Then, using Eq. (19), one obtains

$$
\begin{aligned}
\gamma_{\text {heuristic }}^{\alpha \alpha^{\prime}}\left(E, E^{\prime} ; \omega\right)= & \lambda^{2} \operatorname{tr}_{\mathrm{B}}\left(B^{\alpha^{\prime} \dagger} \Pi_{E} B^{\alpha} \Pi_{E^{\prime}}\right) \\
& \times \int_{\mathbb{R}} d \tau e^{i\left(\omega+E-E^{\prime}\right) \tau} .
\end{aligned}
$$

The macroscopic energies of the bath can always be expressed as $E=n \delta$ for some $n \in \mathbb{N}$. Then the time integrals give rise to

$$
\int_{\mathbb{R}} d(\delta \tau) e^{ \pm i\left[(\omega / \delta)+n-n^{\prime}\right](\delta \tau)} \approx 2 \pi \delta_{E^{\prime}, E+\omega_{k q}}^{\prime},
$$

where the modified Kronecker $\delta$ should be interpreted as the function

$$
\delta_{E^{\prime}, E+\omega_{k q}}^{\prime}=\left\{\begin{array}{cc}
1 & \text { if } E^{\prime} \text { subject to }\left|E^{\prime}-E-\omega\right| \leq \delta \\
0 & \text { otherwise, }
\end{array}\right.
$$


and then one can directly identify the expression $\gamma_{\text {heuristic }}^{\alpha \alpha^{\prime}}\left(E, E^{\prime}\right)$ in Eq. (25).

\section{Details of the RMT approach}

We consider bath coupling operators of the form

$$
B^{\alpha}=\sum_{E \neq E^{\prime}} \sum_{E_{i} \in E_{\delta}} \sum_{E_{j} \in E_{\delta}^{\prime}}\left[b^{\alpha}\left(E, E^{\prime}\right)+c^{\alpha}\left(E_{i}, E_{j}\right)\right]\left|E_{i}\right\rangle\left\langle E_{j}\right|,
$$

where $b^{\alpha}\left(E, E^{\prime}\right)$ are deterministic functions of the macroscopic energies and $c^{\alpha}\left(E_{i}, E_{j}\right)$ are independent and identically distributed complex random numbers with zero mean and variance $\mathbb{E}\left[c^{\alpha}\left(E_{i}, E_{j}\right) c^{\alpha^{\prime}}\left(E_{i}^{\prime}, E_{j}^{\prime}\right)\right]=a^{2} \delta_{\alpha \alpha^{\prime}} \delta_{E_{i} E_{i}^{\prime}} \delta_{E_{j} E_{j}^{\prime}}$. Then the ensemble-averaged bath correlation function yields

$$
\begin{gathered}
\mathbb{E}\left[C_{\mathrm{B}}^{\alpha \alpha^{\prime}}\left(E, E^{\prime} ;-\tau\right)\right]=\frac{\lambda^{2}}{V_{E^{\prime}}}\left[b^{\alpha^{\prime} *}\left(E, E^{\prime}\right) b^{\alpha}\left(E, E^{\prime}\right)+a^{2} \delta_{\alpha \alpha^{\prime}}\right] \\
\times \sum_{E_{i} \in E_{\delta}} \sum_{E_{j} \in E_{\delta}^{\prime}} e^{i E_{i} \tau} e^{-i E_{j} \tau} .
\end{gathered}
$$

To compute the double-sum term in Eq. (A6), we introduce the density of states $g(E)=\partial_{E} \sum_{E_{i}} \Theta\left(E-E_{i}\right)$ and assume (i) the bath is dense enough to justify $\sum_{E_{i}} \mapsto$ $\int g(e) d e$ and (ii) $g(e)$ is approximately constant in each energy window. Then

$$
\sum_{E_{i} \in E_{\delta}} \sum_{E_{j} \in E_{\delta}^{\prime}} e^{i\left(E_{i}-E_{j}\right) \tau} \approx V_{E} V_{E^{\prime}} e^{i\left(E-E^{\prime}\right) \tau} \frac{\sin ^{2}(\delta \tau / 2)}{(\delta \tau / 2)^{2}},
$$

where we have used the relation $g(E) \delta=V_{E}$. The timedependent properties of the correlation function are then described by the function

$$
h(\tau)=\frac{\delta}{2 \pi} \frac{\sin ^{2}(\delta \tau / 2)}{(\delta \tau / 2)^{2}}
$$

As we show in Appendix B, the Fourier transform of $h(\tau)$ is strongly peaked around the origin, and it allows us to approximate

$$
\int_{\mathbb{R}} d \tau h(\tau) e^{i \Omega \tau}=\hat{h}(\Omega) \approx \delta_{\Omega, 0},
$$

where in the computation of the complex dissipation rates, $\Omega=\omega+E-E^{\prime}$. Then, using Eq. (19), we obtain the complex dissipation rates

$$
\begin{aligned}
& \gamma_{\mathrm{RMT}}^{\alpha \alpha^{\prime}}\left(E, E^{\prime}\right) \\
& \quad=\frac{2 \pi \lambda^{2}}{\delta}\left[b_{\alpha^{\prime}}^{*}\left(E, E^{\prime}\right) b_{\alpha}\left(E, E^{\prime}\right)+a^{2} \delta_{\alpha \alpha^{\prime}}\right] V_{E} V_{E^{\prime}} .
\end{aligned}
$$

\section{Details of the ETH approach}

To use the ETH to compute the correlation function in Eq. (A1), two issues arise: first, there is no guarantee that the operators $B^{\alpha}$ are Hermitian and, second, it is not clear how the ETH should be modified when one considers correlation between different observables. The first issue is easily solved by noting that any operator can be decomposed as $O=O_{+}+i O_{-}$, where $O_{+}$and $O_{-}$are Hermitian. Therefore,

$$
V=\lambda \sum_{\alpha} S^{\alpha} \otimes B^{\alpha}=\lambda \sum_{\alpha}\left(S_{+}^{\alpha} \otimes B_{+}^{\alpha}-S_{-}^{\alpha} \otimes B_{-}^{\alpha}\right) .
$$

Then we can assume without loss of generality that $S^{\alpha}$ and $B^{\alpha}$ are Hermitian. Then, using the ETH ansatz, we find

$$
B_{i j}^{\alpha}=B^{\alpha}\left(E_{i j}\right) \delta_{i j}+\sqrt{\frac{1}{V_{E_{i j}}}} f^{\alpha}\left(E_{i j}, \Omega_{i j}\right) R_{i j}^{\alpha} .
$$

Because the definition of $B^{\alpha}$ is such that $\left\langle B^{\alpha}\right\rangle_{E}=0$ for all $E$, the first term of the ansatz can be set to zero. Then the microcanonical bath correlation function yields

$$
\begin{aligned}
& C_{\mathrm{B}}^{\alpha \alpha^{\prime}}\left(E, E^{\prime} ;-\tau\right) \\
& \quad=\frac{\lambda^{2}}{V_{E^{\prime}}} \sum_{E_{i}, E_{j}} \frac{f^{\alpha^{\prime} *}\left(E_{i j}, \Omega_{i j}\right) f^{\alpha}\left(E_{i j}, \Omega_{i j}\right)}{V_{E_{i j}}} R_{i j}^{\alpha^{\prime} *} R_{i j}^{\alpha} e^{i\left(E_{i}-E_{j}\right) \tau} .
\end{aligned}
$$

Now we would like to use the statistical properties of the erratically varying random numbers $R_{i j}^{\alpha}$. It is clear that $R_{i j}^{\alpha}$ should have zero mean as before. However, $R_{i j}^{\alpha}$ cannot be uncorrelated for different $\alpha$ or otherwise two-point correlation of two generic different observables would vanish [28]. Thus, we proceed without assigning a value to the correlation of $\overline{R_{i j}^{\alpha^{\prime *} *} R_{i j}^{\alpha}}$, where here the overline denotes the average in the spirit of the ETH.

We proceed as follows: On one hand, the numbers $R_{i j}^{\alpha}$ change erratically with $i$ and $j$ and even $j \mapsto j+1$ can abruptly change its value. On the other hand, the function $f^{\alpha}(E, \Omega)$ is a smooth function of its arguments and, for a dense enough bath, the substitution $j \mapsto j+1$ will give rise to a perturbative correction. Therefore, it is justified to substitute in Eq. (A13) $R_{i j}^{\alpha^{\prime} *} R_{i j}^{\alpha} \mapsto \overline{R_{i j}^{\alpha^{\prime} *} R_{i j}^{\alpha}}$. Then we introduce the function

$$
F^{\alpha \alpha^{\prime}}\left(E_{i j}, \Omega_{i j}\right):=f^{\alpha^{\prime} *}\left(E_{i j}, \Omega_{i j}\right) f^{\alpha}\left(E_{i j}, \Omega_{i j}\right) \overline{R_{i j}^{\alpha^{\prime} *} R_{i j}^{\alpha}},
$$

which, consistently with the ETH ansatz, is a smooth function of its arguments, decays with $|\Omega| \rightarrow \infty$, and has the 
symmetry property

$$
\left[F^{\alpha \alpha^{\prime}}(E, \Omega)\right]^{*}=F^{\alpha^{\prime} \alpha}(E, \Omega)=F^{\alpha \alpha^{\prime}}(E,-\Omega) .
$$

For a sufficiently regular and dense environment, it is justified to approximate $E_{i j} \approx \bar{E}=\left(E+E^{\prime}\right) / 2$ and replace $\sum_{E_{i}} \mapsto \int \operatorname{deg}(e)$. Assuming that $g(e)$ is constant within each energy window, we arrive at

$$
\begin{aligned}
& C_{\mathrm{B}}^{\alpha \alpha^{\prime}}\left(E, E^{\prime} ;-\tau\right)=\lambda^{2} \frac{g(E) g\left(E^{\prime}\right)}{V_{E^{\prime}}} \\
& \quad \times \iint d e d e^{\prime} \frac{F^{\alpha \alpha^{\prime}}\left(\bar{E}, e-e^{\prime}\right)}{V_{\bar{E}}} e^{i\left(e-e^{\prime}\right) \tau},
\end{aligned}
$$

Finally, using Eq. (19), we find the integration over time of the correlation function gives a factor $2 \pi \delta\left(\omega+e-e^{\prime}\right)$ and leads to the expression

$$
\gamma_{\mathrm{ETH}}^{\alpha \alpha^{\prime}}\left(E, E^{\prime}\right)=\frac{2 \pi \lambda^{2}}{\delta} V_{E} V_{E^{\prime}} \frac{F^{\alpha \alpha^{\prime}}\left(\bar{E}, E-E^{\prime}\right)}{V_{\bar{E}}} .
$$

\section{Connection between the three approaches}

Finally, we investigate under what circumstances the three derivations given above give rise to the same decay rates. To this end, we rewrite them in the alternative form

$$
\begin{aligned}
& \gamma_{\text {heuristic }}^{\alpha \alpha^{\prime}}\left(E, E^{\prime}\right)=\frac{2 \pi \lambda^{2}}{\delta} V_{E} V_{E^{\prime}} \frac{\operatorname{tr}\left(B_{\alpha^{\prime}}^{\dagger} \Pi_{E} B_{\alpha} \Pi_{E^{\prime}}\right)}{V_{E} V_{E^{\prime}}}, \\
& \gamma_{\mathrm{RMT}}^{\alpha \alpha^{\prime}}\left(E, E^{\prime}\right)=\frac{2 \pi \lambda^{2}}{\delta} V_{E} V_{E^{\prime}}\left[b_{\alpha^{\prime}}^{*}\left(E, E^{\prime}\right) b_{\alpha}\left(E, E^{\prime}\right)+a^{2} \delta_{\alpha \alpha^{\prime}}\right], \\
& \gamma_{\mathrm{ETH}}^{\alpha \alpha^{\prime}}\left(E, E^{\prime}\right)=\frac{2 \pi \lambda^{2}}{\delta} V_{E} V_{E^{\prime}} \frac{F^{\alpha \alpha^{\prime}}\left(\bar{E}, E-E^{\prime}\right)}{V_{\bar{E}}}
\end{aligned}
$$

which makes the comparison easier. A connection can be found when the coupling has purely coarse-grained components; namely, when the following approximation holds:

$$
\begin{aligned}
B^{\alpha} & =\sum_{E_{i} E_{j}}\left\langle E_{i}\left|B^{\alpha}\right| E_{j}\right\rangle\left|E_{i}\right\rangle\left\langle E_{j}\right| \\
& \approx \sum_{E E^{\prime}} b^{\alpha}\left(E, E^{\prime}\right) \sum_{E_{i} \in E_{\delta}} \sum_{E_{j} \in E_{\delta}^{\prime}}\left|E_{i}\right\rangle\left\langle E_{j}\right|,
\end{aligned}
$$

where $b^{\alpha}\left(E, E^{\prime}\right)$ are functions of only the coarse grained energies.

Connection of the approaches in Secs. II C 1 and II C 2

Assuming the form in Eq. (A19), the coarse bath dynamics method gives raise to the rates

$$
\gamma_{\text {heuristic }}^{\alpha \alpha^{\prime}}\left(E, E^{\prime}\right)=\frac{2 \pi \lambda^{2}}{\delta} V_{E} V_{E^{\prime}} b_{\alpha^{\prime}}^{*}\left(E, E^{\prime}\right) b_{\alpha}\left(E, E^{\prime}\right)
$$

On the other hand, in the limit of vanishing variance $a \rightarrow$ 0 , the random matrix coupling in Eq. (26) reduces to (A19).
Therefore, we find

$$
\begin{aligned}
& \gamma_{\mathrm{RMT}}^{\alpha \alpha^{\prime}}\left(E, E^{\prime}\right) \\
& \quad=\lim _{a \rightarrow 0} \frac{2 \pi \lambda^{2}}{\delta} V_{E} V_{E^{\prime}}\left[b_{\alpha^{\prime}}^{*}\left(E, E^{\prime}\right) b_{\alpha}\left(E, E^{\prime}\right)+a^{2} \delta_{\alpha \alpha^{\prime}}\right] \\
& \quad=\frac{2 \pi \lambda^{2}}{\delta} V_{E} V_{E^{\prime}} b_{\alpha^{\prime}}^{*}\left(E, E^{\prime}\right) b_{\alpha}\left(E, E^{\prime}\right),
\end{aligned}
$$

obtaining then the same rates $\gamma_{\text {heuristic }}^{\alpha \alpha^{\prime}}\left(E, E^{\prime}\right)=\gamma_{\mathrm{RMT}}^{\alpha \alpha^{\prime}}$ $\left(E, E^{\prime}\right)$.

Connection of the approaches in Secs. II C 1 and II C 3

Using the ETH ansatz in Eq. (A12) and assuming that the bath coupling operator has the coarse-grained structure in Eq. (A19), we find

$$
B_{i j}^{\alpha}=\sqrt{\frac{1}{V_{\bar{E}}}} f^{\alpha}\left(\bar{E}, E-E^{\prime}\right) R_{i j}^{\alpha} \quad\left(E \neq E^{\prime}\right) .
$$

Using Eq. (A22) in Eq. (25) and identifying the average $\overline{R_{\alpha^{\prime}, i j}^{*} R_{\alpha, i j}}=\sum_{i j} R_{\alpha^{\prime}, i j}^{*} R_{\alpha, i j} /\left(V_{E} V_{E^{\prime}}\right)$, we arrive at

$$
\gamma_{\text {heuristic }}^{\alpha \alpha^{\prime}}\left(E, E^{\prime}\right)=\frac{2 \pi \lambda^{2}}{\delta} V_{E} V_{E^{\prime}} \frac{F^{\alpha \alpha^{\prime}}\left(\bar{E}, E-E^{\prime}\right)}{V_{\bar{E}}},
$$

and therefore $\gamma_{\text {heuristic }}^{\alpha \alpha^{\prime}}\left(E, E^{\prime}\right)=\gamma_{\mathrm{ETH}}^{\alpha \alpha^{\prime}}\left(E, E^{\prime}\right)$.

\section{APPENDIX B: MORE DETAILS ON THE RMT APPROACH}

Here we give further details on the RMT approach. Our starting point is the finite-time Redfield version of the EMME, which in the Schrödinger picture reads

$$
\begin{gathered}
\partial_{t} \rho_{\mathrm{S}}(E)=-i\left[\mathrm{H}_{\mathrm{S}}, \rho_{\mathrm{S}}(E)\right]+\sum_{\alpha \alpha^{\prime}} \sum_{E^{\prime}} \sum_{\omega \omega^{\prime}} \int_{0}^{t} d \tau e^{-i \omega^{\prime} \tau} \\
\times\left\{\mathbb{E}\left[C_{\mathrm{B}}^{\alpha \alpha^{\prime}}\left(E, E^{\prime} ;-\tau\right)\right] S_{\omega} \rho_{\mathrm{S}}\left(E^{\prime}\right) S_{\omega^{\prime}}^{\dagger}\right. \\
\left.-\mathbb{E}\left[C_{\mathrm{B}}^{\alpha \alpha^{\prime}}\left(E^{\prime}, E ;-\tau\right)\right] \rho_{\mathrm{S}}(E) S_{\omega^{\prime}}^{\dagger} S_{\omega}\right\}+ \text { h.c.. }
\end{gathered}
$$

Our goal consists in evaluating more explicitly the integrals of the form

$$
\int_{0}^{t} d \tau \mathbb{E}\left[C_{\mathrm{B}}^{\alpha \alpha^{\prime}}\left(E, E^{\prime} ;-\tau\right)\right] e^{i \omega \tau}
$$

which appear in Eq. (B1). To that end, we use the results obtained in Appendix A 2 to write the averaged microcanonical bath correlation function as

$$
\mathbb{E}\left[C_{\mathrm{B}}^{\alpha \alpha^{\prime}}\left(E, E^{\prime} ;-\tau\right)\right]=\frac{\gamma_{\mathrm{RMT}}^{\alpha \alpha^{\prime}}\left(E, E^{\prime}\right)}{V_{E^{\prime}}} h(\tau) e^{i\left(E-E^{\prime}\right) \tau} .
$$


Then the integrals of interest can be cast as

$$
\frac{\gamma_{\mathrm{RMT}}^{\alpha \alpha^{\prime}}\left(E, E^{\prime}\right)}{V_{E^{\prime}}} \int_{0}^{t} d \tau h(\tau) e^{i\left(\omega+E-E^{\prime}\right) \tau}
$$

In the case where $\omega+E=E^{\prime}$, the evaluation is simpler and can be done by conveniently introducing the function

$$
\zeta(t):=\frac{\delta}{\pi} \int_{0}^{t} d \tau \frac{\sin ^{2}(\delta \tau / 2)}{(\delta \tau / 2)^{2}}=2 \int_{0}^{t} d \tau h(\tau)
$$

Then all the dissipation rates appearing in the EMME are multiplied by the time-dependent envelope $\zeta(t)$ as is the case, for instance, in the example analyzed in Sec. IV.

In general, however, there is no a priori reason why $E^{\prime}=E+\omega$. Even though the general result for a finite $t$ is cumbersome, it is possible to evaluate exactly the time integrals under the Markov approximation (i.e., for $t \rightarrow$ $\infty)$. Ultimately, our objective is to compute the integrals

$$
I(1):=\int_{0}^{\infty} d \tau h(\tau) e^{-i \Omega \tau}=\frac{1}{\pi} \int_{0}^{\infty} d x \frac{\sin ^{2} x}{x^{2}} e^{-i 2(\Omega / \delta) x},
$$

which appear in Eq. (B4). Those integrals can be regarded as the Laplace transform $\breve{h}(i \Omega)=\mathbb{L}[h(\tau)](s=i \Omega)$, with $\mathbb{L}[f(\tau)](s)=\int_{0}^{\infty} d \tau f(\tau) \exp (-s \tau)$. It is easy to see that the real and imaginary parts of $\breve{h}(i \Omega)$ correspond respectively to even and odd functions of $\Omega$. We introduce the frequency ratio $\xi=\Omega / \delta \in \mathbb{R}$ as well as the parameterdependent integral

$$
\begin{aligned}
I(a) & :=\frac{1}{\pi} \int_{0}^{\infty} d x \frac{\sin ^{2} a x}{x^{2}} e^{-i 2 \xi x} \\
& \Rightarrow I^{\prime}(a)=\frac{1}{\pi} \int_{0}^{\infty} 2 a d x \frac{\sin 2 a x}{2 a x} e^{-i 2 \xi x} .
\end{aligned}
$$

Performing the change of variables $t=2 a x$ and noting the Laplace transform property

$$
\begin{aligned}
& \mathbb{L}[f(t) / t](s)=\int_{s}^{\infty} d u \breve{f}(u) \\
& \quad \Rightarrow \mathbb{L}(\sin t / t)(s)=\int_{s}^{\infty} \frac{d u}{1+u^{2}}=\frac{\pi}{2}-\arctan s,
\end{aligned}
$$

we obtain $I^{\prime}(a)=1 / 2-\arctan (i \xi / a) / \pi$. The complex function $w(z)=\arctan z$ can now be written in terms of logarithms using the following reasoning:

$$
\begin{aligned}
z & =\tan w=-i \frac{e^{i w}-e^{-i w}}{e^{i w}+e^{-i w}} \\
& \Rightarrow 2 i w=\log \frac{1+i z}{1-i z}+n 2 \pi i, \quad \text { with } n \in \mathbb{N} .
\end{aligned}
$$

We choose the principal Riemann sheet $n=0$ to coincide with the real arctan function; that is, $w(1)=\pi / 4$. Noticing that $I(0)=0$, we can proceed to

$$
\begin{aligned}
I(1) & =\int_{0}^{1} d a I^{\prime}(a)=\int_{0}^{1} d a\left(\frac{1}{2}-\frac{\arctan (i \xi / 2)}{\pi}\right) \\
& =\frac{1}{2}+\frac{i}{2 \pi} \int_{0}^{1} d a[\log (a-\xi)-\log (a+\xi)] .
\end{aligned}
$$

As we have discussed, the real and imaginary parts of the target integral $I(1)$ are respectively even and odd functions of $\xi$, and then we can restrict ourselves to $\xi>0$. Still, we have two different scenarios:

$$
\begin{aligned}
& \int_{0}^{1} d a[\log (a+\xi)-\log (a-\xi)]=\int_{0}^{1} \log (a+\xi) \\
& -\int_{0}^{\xi} d a \log (a-\xi)-\int_{\xi}^{1}(\log |a-\xi|+i \pi)(\text { for } \xi<1), \\
& \int_{0}^{1} d a[\log (a+\xi)-\log (a-\xi)]=\int_{0}^{1} \log (a+\xi) \\
& \quad+\int_{0}^{1}(\log |a-\xi|+i \pi) \quad(\text { for } \xi>1),
\end{aligned}
$$

which leads, for $\xi>0$, to the final result

$$
\begin{aligned}
\operatorname{Re}[I(1)]= & \left\{\begin{array}{cc}
(1-\xi) / 2 & \text { for } \xi<1, \\
0 & \text { for } \xi>1,
\end{array}\right. \\
\operatorname{Im}[I(1)]= & \frac{1}{2 \pi}[2 \xi \log (\xi)-(1+\xi) \log (1+\xi) \\
& +(1-\xi) \log |1-\xi|] .
\end{aligned}
$$

Therefore, there is no approximation in $\operatorname{Re}[\breve{h}(i \Omega)]$ by disregarding the nonresonant terms $\Omega>\delta$. For completeness we show the full behavior in Fig. 6. Hence, the

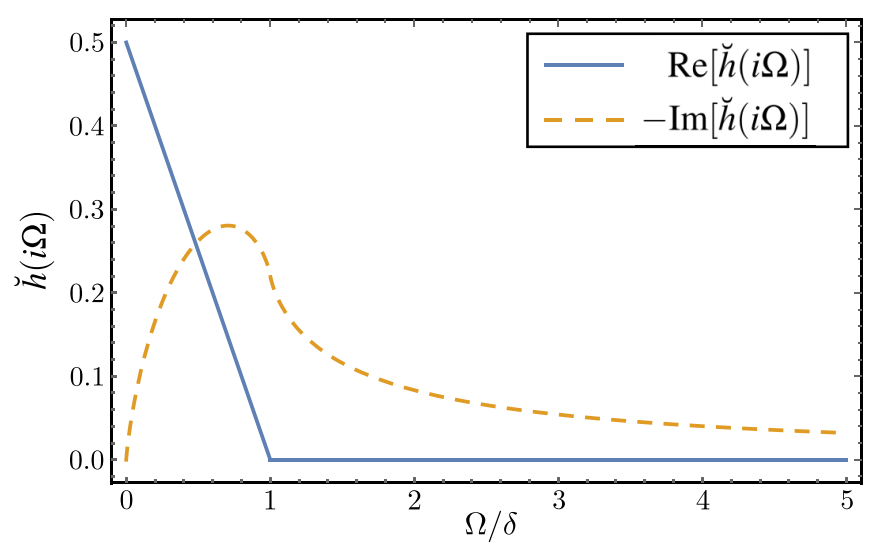

FIG. 6. Real (solid blue line) and imaginary (dashed orange line) parts of the function $\breve{h}(i \Omega)$. 
relation $\gamma_{\mathrm{RMT}}^{\alpha \alpha^{\prime}}\left(E, E^{\prime} ; \omega\right) \propto \delta_{E^{\prime}, E+\omega}$ is obtained without any approximation in computing the time integrals.

\section{APPENDIX C: PROPERTIES OF THE TRANSTION RATES}

Here we consider the interaction $\mathrm{H}_{\text {int }}=\lambda \sum_{\alpha} S^{\alpha} \otimes B_{\text {int }}^{\alpha}$ leading to the transition rates

$$
W_{k q}\left(E, E^{\prime}\right):=\sum_{\alpha \alpha^{\prime}}\left\langle q\left|S^{\alpha^{\prime} \dagger}\right| k\right\rangle\left\langle k\left|S^{\alpha}\right| q\right\rangle \gamma^{\alpha \alpha^{\prime}}\left(E, E^{\prime}\right)
$$

With this definition, we prove the properties $W_{k q}\left(E, E^{\prime}\right) \geq$ 0 and $W_{k q}\left(E, E^{\prime}\right)=W_{q k}\left(E^{\prime}, E\right)$ for the transition rates. The former is proven, as usual, through Bochner's theorem and the latter requires only basic algebraic manipulation.

\section{Positivity}

We start by noting that the transition rates $W_{k q}\left(E, E^{\prime}\right)$ may be obtained from the scalar product

$$
\left(\mathbf{s}_{k q}, \gamma^{T} \mathbf{s}_{k q}\right)=\sum_{\alpha \alpha^{\prime}} s_{k q}^{\alpha^{\prime} *} \gamma^{\alpha \alpha^{\prime}}\left(E, E^{\prime} ; \omega\right) s_{k q}^{\alpha}
$$

where the arguments $E, E^{\prime}$ and $\omega$ are implicit in the matrix $\gamma^{T}$. Defining $V_{k q}=\lambda \sum_{\alpha} s_{k q}^{\alpha} B^{\alpha}$, we find Eq. (C2) is the Fourier transform

$$
\begin{aligned}
\left(\mathbf{s}_{k q}, \gamma^{T} \mathbf{s}_{k q}\right) & \left.=\int_{\mathbb{R}} d \tau \operatorname{tr}_{\mathrm{B}}\left(e^{-i \mathrm{H}_{\mathrm{B}} \tau} V_{k q}^{\dagger} e^{i \mathrm{H}_{\mathrm{B}} \tau} \Pi_{E} V_{k q} \Pi_{E^{\prime}}\right]\right) e^{i \omega \tau} \\
& =\int_{\mathbb{R}} d \tau f(\tau) e^{i \omega \tau}=\hat{f}(\omega) .
\end{aligned}
$$

Bochner's theorem states that the Fourier transform $\hat{f}(\omega)$ of a function $f(\tau)$ is positive if $f(\tau)$ is of positive type. A function is of positive type if for any set of times $\left\{\tau^{\alpha}\right\}$ the matrix $f^{\alpha \alpha^{\prime}} \equiv f\left(\tau^{\alpha}-\tau^{\alpha^{\prime}}\right)$ is positive semidefinite. Then we see that $f(\tau)$ is of positive type since taking a general vector $\mathbf{w}$, we have

$$
\begin{aligned}
(\mathbf{w}, \mathbf{f w}) & =\sum_{E_{i} E_{j}}\left|\sum_{\alpha} w^{\alpha} e^{-i\left(E_{i}-E_{j}\right) \tau^{\alpha}}\right|^{2}\left|\left\langle E_{i}\left|V_{k q}\right| E_{j}\right\rangle\right|^{2} \\
& \geq 0
\end{aligned}
$$

where $E_{i} \in E_{\delta}$ and $E_{j} \in E_{\delta}^{\prime}$. Finally, to obtain $W_{k q}\left(E, E^{\prime}\right) \geq$ 0 from the inner product $\left(\mathbf{s}_{k q}, \gamma^{T} \mathbf{s}_{k q}\right) \geq 0$, it is only left to assume the factorization condition, which yields

$$
W_{k q}\left(E, E^{\prime}\right) \delta_{E^{\prime}, E+\omega}=\left(\mathbf{s}_{k q}, \gamma^{T} \mathbf{s}_{k q}\right) \geq 0
$$

\section{Symmetry}

The key observation is that since the interaction $V$ is Hermitian, we have the property $V_{k q}^{\dagger}=V_{q k}$. Then one can cast Eq. (C3) in the alternative form

$$
\left(\mathbf{s}_{k q}, \gamma^{T} \mathbf{s}_{k q}\right)=\sum_{E_{i} E_{j}}\left|\left\langle E_{i}\left|V_{k q}\right| E_{j}\right\rangle\right|^{2} \int_{\mathbb{R}} d \tau e^{i\left(\omega+E_{i}-E_{j}\right) \tau},
$$

where $E_{i} \in E_{\delta}$ and $E_{j} \in E_{\delta}^{\prime}$. From the equation above, one deduces

$$
\left[\mathbf{s}_{k q}, \gamma^{T}\left(E, E^{\prime} ; \omega\right) \mathbf{s}_{k q}\right]=\left[\mathbf{s}_{q k}, \gamma^{T}\left(E^{\prime}, E ;-\omega\right) \mathbf{s}_{q k}\right]
$$

Therefore, assuming the factorization condition in Eq. (6), it follows that

$$
W_{k q}\left(E, E^{\prime}\right) \delta_{E^{\prime}, E+\omega}=W_{q k}\left(E^{\prime}, E\right) \delta_{E, E^{\prime}-\omega},
$$

from which we deduce $W_{k q}\left(E, E^{\prime}\right)=W_{q k}\left(E^{\prime}, E\right)$.

\section{APPENDIX D: MUTUAL INFORMATION INEQUALITY}

Here we prove the inequality $\mathcal{I}^{\mathrm{S}: \mathrm{B}}[\rho] \geq \mathcal{I}_{\mathrm{CG}}^{\mathrm{S}: \mathrm{B}}(\mathbf{p})$. First, we note that the quantum mutual information can be cast as $\mathcal{I}^{\mathrm{S}: \mathrm{B}}[\rho]=\mathcal{D}\left[\rho \| \rho_{\mathrm{S}} \otimes \rho_{\mathrm{B}}\right]$, where we have introduced the quantum relative entropy

$$
\mathcal{D}[\rho \| \sigma]=\operatorname{tr}[\rho(\log \rho-\log \sigma)] \geq 0
$$

for two states. $\rho$ and $\sigma$ The relative entropy is contractive under the action of a completely positive and tracepreserving map $\mathcal{E}$; that is, it fulfills the property $\mathcal{D}[\rho \| \sigma] \geq$ $\mathcal{D}[\mathcal{E}(\rho) \| \mathcal{E}(\sigma)]$. Second, we note that the map

$$
\begin{aligned}
\mathcal{E}[\rho] & =\sum_{k, E} \operatorname{tr}\left[\rho|k\rangle\langle k| \otimes \Pi_{E}\right]|k\rangle\langle k| \otimes \frac{\Pi_{E}}{V_{E}} \\
& =\sum_{k, E} p\left(\varepsilon_{k}, E\right)|k\rangle\langle k| \otimes \frac{\Pi_{E}}{V_{E}},
\end{aligned}
$$

is a valid completely positive and trace-preserving map since its Kraus decomposition can be read from

$$
\mathcal{E}[\rho]=\sum_{k, E} \sum_{E_{i} \in E_{\delta}} \sum_{E_{j} \in E_{\delta}} \frac{\left|k, E_{i}\right\rangle\left\langle k, E_{j}\right|}{\sqrt{V_{E}}} \rho \frac{\left|k, E_{j}\right\rangle\left\langle k, E_{i}\right|}{\sqrt{V_{E}}} .
$$

Therefore, we obtain

$$
\begin{aligned}
\mathcal{I}^{\mathrm{S}: \mathrm{B}}[\rho] & =\mathcal{D}\left[\rho \| \rho_{\mathrm{S}} \otimes \rho_{\mathrm{B}}\right] \\
& \geq \mathcal{D}\left[\mathcal{E}[\rho] \| \mathcal{E}\left[\rho_{\mathrm{S}} \otimes \rho_{\mathrm{B}}\right]\right]=\mathcal{I}_{\mathrm{CG}}^{\mathrm{S}: \mathrm{B}}(\mathbf{p}) .
\end{aligned}
$$




\section{APPENDIX E: ANALYTICAL SOLUTION FOR THE SPIN SYSTEM}

Here we provide the analytical solution for the spin system studied in Sec. IV. Taking as a starting point Eq. (45), we project into the system eigenstates, from which we obtain the population rate equations

$$
\begin{aligned}
\partial_{t} p\left(\varepsilon_{0}, E\right)= & \zeta(t) \gamma(E, E-\Delta \varepsilon) \\
& \times\left(\frac{p\left(\varepsilon_{1}, E-\Delta \varepsilon\right)}{V_{E-\Delta \varepsilon}}-\frac{p\left(\varepsilon_{0}, E\right)}{V_{E}}\right), \\
\partial_{t} p\left(\varepsilon_{1}, E\right)= & \zeta(t) \gamma(E, E+\Delta \varepsilon) \\
& \times\left(\frac{p\left(\varepsilon_{0}, E+\Delta \varepsilon\right)}{V_{E+\Delta \varepsilon}}-\frac{p\left(\varepsilon_{1}, E\right)}{V_{E}}\right),
\end{aligned}
$$

where we have defined $\gamma\left(E, E^{\prime}\right)=0$ if either $E$ or $E^{\prime}$ does not exist. Equivalently, we could have written Eq. (E1) in matrix form by gathering all the populations $p\left(\varepsilon_{k}, E\right)$ in the population vector $\mathbf{p}$ as

$$
\partial_{t} \mathbf{p}=\zeta(t) \Lambda \mathbf{p},
$$

where the entries of the matrix $\Lambda$ should be read from Eq. (E1) (see below). Equation (E1) leads to the blockdiagonal structure

$$
\Lambda=\bigoplus_{E_{\mathrm{tot}}} \Lambda\left(E_{\mathrm{tot}}\right)
$$

where $\Lambda\left(E_{\text {tot }}\right)$ acts on the subspace $\mathbf{p}\left(E_{\text {tot }}\right)=\left\{p\left(\varepsilon_{1}, E\right)\right.$, $\left.p\left(\varepsilon_{0}, E+\Delta \varepsilon\right)\right\}$ of total energy $E_{\text {tot }}=\varepsilon_{1}+E$. Explicitly, we can write

$$
\Lambda\left(E_{\mathrm{tot}}\right)=\gamma(E, E+\Delta \varepsilon)\left(\begin{array}{cc}
-1 / V_{E} & 1 / V_{E+\Delta \varepsilon} \\
1 / V_{E} & -1 / V_{E+\Delta \varepsilon}
\end{array}\right)
$$

which is a stochastic matrix. Now we are in the position where it is possible to integrate Eq. (E2) to arrive at

$$
\mathbf{p}(t)=\bigoplus_{E_{\mathrm{tot}}} e^{\Lambda\left(E_{\mathrm{tot}}\right) \Xi(t)} \mathbf{p}(0),
$$

where $\Xi(t)=\int_{0}^{t} d t^{\prime} \zeta\left(t^{\prime}\right)$. Remarkably the matrix $\Lambda(E)$ has the property

$$
\begin{aligned}
\Lambda\left(E_{\mathrm{tot}}\right)^{2} & =-\gamma(E, E+\Delta \varepsilon)\left(\frac{1}{V_{E}}+\frac{1}{V_{E+\Delta \varepsilon}}\right) \Lambda\left(E_{\mathrm{tot}}\right) \\
& \equiv-2 \bar{\gamma}(E, E+\Delta \varepsilon) \Lambda\left(E_{\mathrm{tot}}\right),
\end{aligned}
$$

which leads to the final solution

$$
\begin{gathered}
e^{\Lambda\left(E_{\mathrm{tot}}\right) \Xi(t)} \mathbf{p}\left(E_{\mathrm{tot}} ; 0\right)=\sum_{n=0}^{\infty} \frac{\left[\Lambda\left(E_{\mathrm{tot}}\right) \Xi(t)\right]^{n}}{n !} \mathbf{p}\left(E_{\mathrm{tot}} ; 0\right) \\
=\left(1+\frac{1-e^{-2 \bar{\gamma}(E, E+\Delta \varepsilon) \Xi(t)}}{2 \bar{\gamma}(E, E+\Delta \varepsilon)} \Lambda\left(E_{\mathrm{tot}}\right)\right) \mathbf{p}\left(E_{\mathrm{tot}} ; 0\right) .
\end{gathered}
$$

Since the function $\zeta(t)$ saturates rapidly to $\zeta(t \rightarrow \infty)=$ 1 , we expect $\Xi(t) \sim t$ at long times. Therefore, the steady state can be computed

$$
\frac{p_{\mathrm{eq}}\left(\varepsilon_{1}, E\right)}{p_{\mathrm{eq}}\left(\varepsilon_{0}, E+\Delta \varepsilon\right)}=\frac{V_{E}}{V_{E+\Delta \varepsilon}},
$$

while we keep constant at all times the probability of being in the energy shell $E_{\text {tot }}=\varepsilon_{1}+E$; that is, $p\left(\varepsilon_{1}, E\right)+$ $p\left(\varepsilon_{0}, E+\Delta \varepsilon\right)=$ constant.

\section{APPENDIX F: ADDITIONAL NUMERICAL RESULTS}

Here we show the evolution of the population for stronger and weaker coupling strengths as compared with the main text (Fig. 2). First, in the first row in Figs. 7-9, we observe that reducing the volumes of the energy bands $V_{E} \lesssim 100$ typically leads to a disagreement of the exact dynamics with the prediction of the EMME. This expected behavior arises from the fact that the bath is too small: recurrences are unavoidable and no perturbative master equation approach can correctly capture the reduced system dynamics in this case.
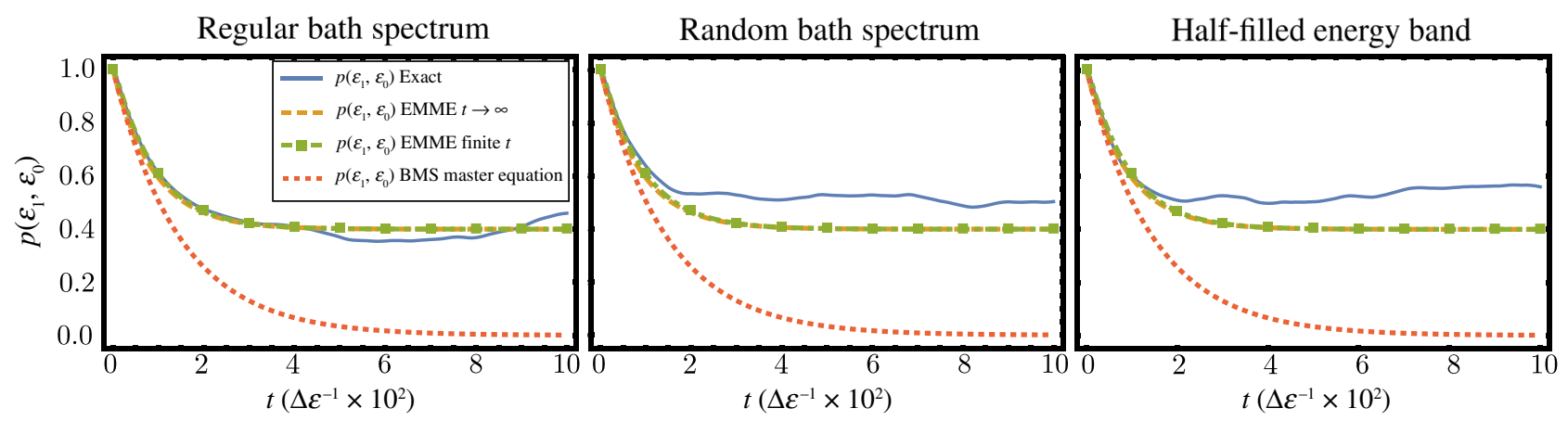

FIG. 7. Comparison of the evolution of the joint probability $p\left(\varepsilon_{1}, E=\varepsilon_{0}\right)$ for a spin system coupled to a two-band bath (see Fig. 2 for details). $\lambda=3 \times 10^{-3}, \delta=0.5, \varepsilon_{0}=0, \varepsilon_{1}=1, V_{\varepsilon_{0}}=20, V_{\varepsilon_{1}}=40$, and $a=1$. 


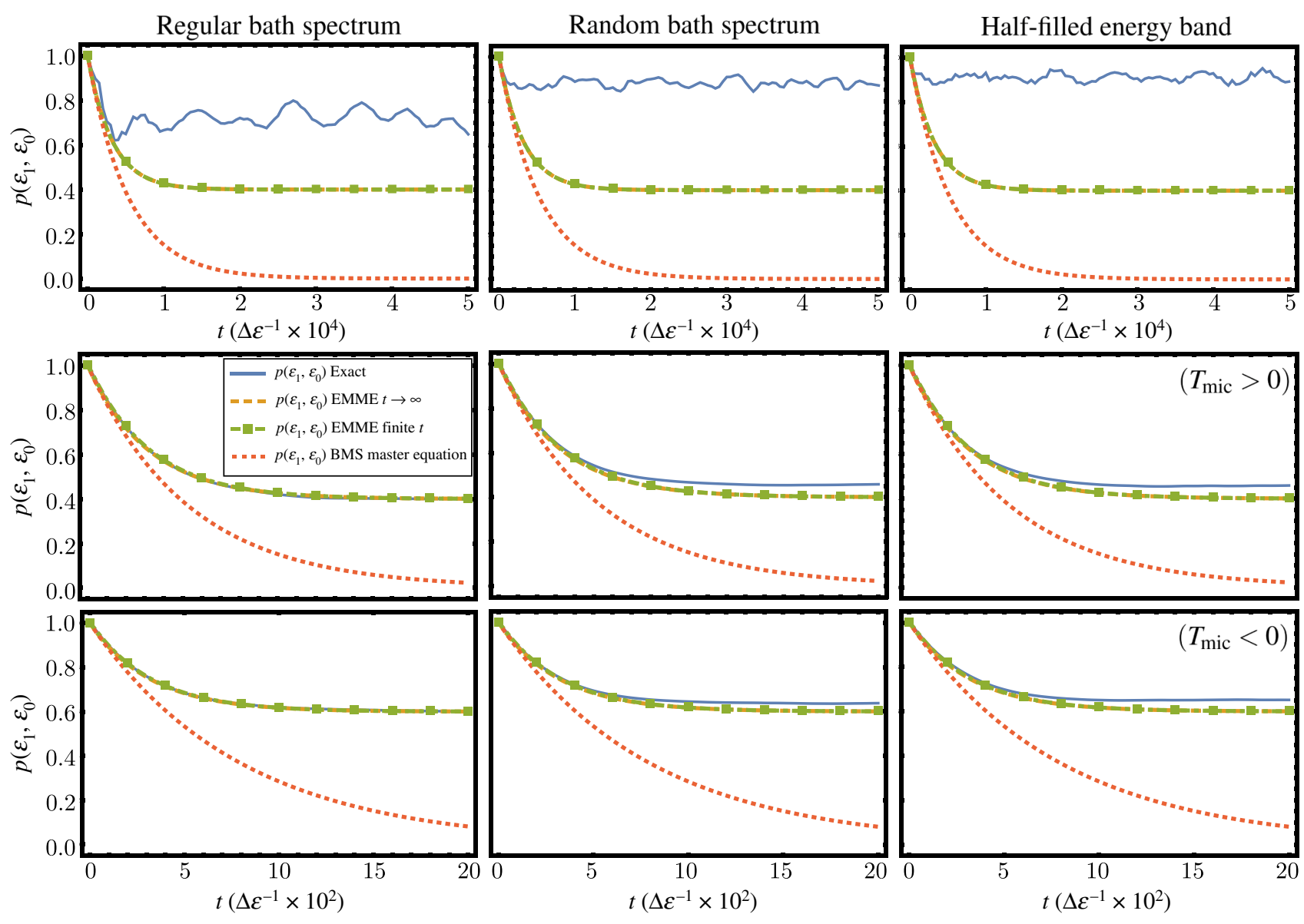

FIG. 8. Comparison of the evolution of the joint probability $p\left(\varepsilon_{1}, E=\varepsilon_{0}\right)$ for a spin system coupled to a two-band bath (see Fig. 2 for details). The first row corresponds to $V_{\varepsilon_{0}}=20$ and $V_{\varepsilon_{1}}=40$. The second row corresponds to $V_{\varepsilon_{0}}=400$ and $V_{\varepsilon_{1}}=600$. The third row corresponds to $V_{\varepsilon_{0}}=600$ and $V_{\varepsilon_{1}}=400 . \lambda=5 \times 10^{-4}, \delta=0.5, \varepsilon_{0}=0, \varepsilon_{1}=1$, and $a=1$.

Regarding the second and third rows in Fig. 8, we observe that reducing the coupling strength $\lambda$ further can lead to imprecise results even though the second-order approximation becomes more accurate. The underlying reason is that as $\lambda \rightarrow 0$, the linewidth of a jump process becomes narrower, and then the system can resolve the fine structure of the bath energy bands. Typically, resolving the structure of the bath energy bands leads to a reduced effective volume of the band or, for a very small number of available levels, may avoid thermalization completely.

In the opposite limit of a "large" coupling $\lambda$ (see Fig. 9), the EMME fails to describe the transient behavior, while the steady state is correctly predicted. Of course, for even larger $\lambda$, the second-order approximation breaks down completely, leading to an incorrect description of the dynamics.

The aforementioned observations are in unison with the findings in Ref. [9], where upper and lower bounds for the coupling strength were found to the validity of the microcanonical master equation.

\section{APPENDIX G: DETAILS ON MULTIPLE ENVIRONMENTS}

Here we show in some detail the derivation of the EMME for the case of multiple baths and, also, we obtain the nonequilibrium thermodynamic description in a manner analogous to the case of a single bath. We generalize the results shown in the main text to a coupling operator of the form $\mathrm{H}_{\mathrm{int}, v}=\sum_{\alpha_{\nu}} S_{v}^{\alpha_{\nu}} \otimes B_{\text {int, },}^{\alpha_{v}}$.

\section{Additive structure}

Following the derivation for a single bath, we consider that each bath is coupled to the system via the interaction operator

$$
\mathrm{H}_{\mathrm{int}, \nu}=\lambda \sum_{\alpha_{\nu}} S_{\nu}^{\alpha_{\nu}} \otimes B_{\mathrm{int}, \nu}^{\alpha_{\nu}} .
$$



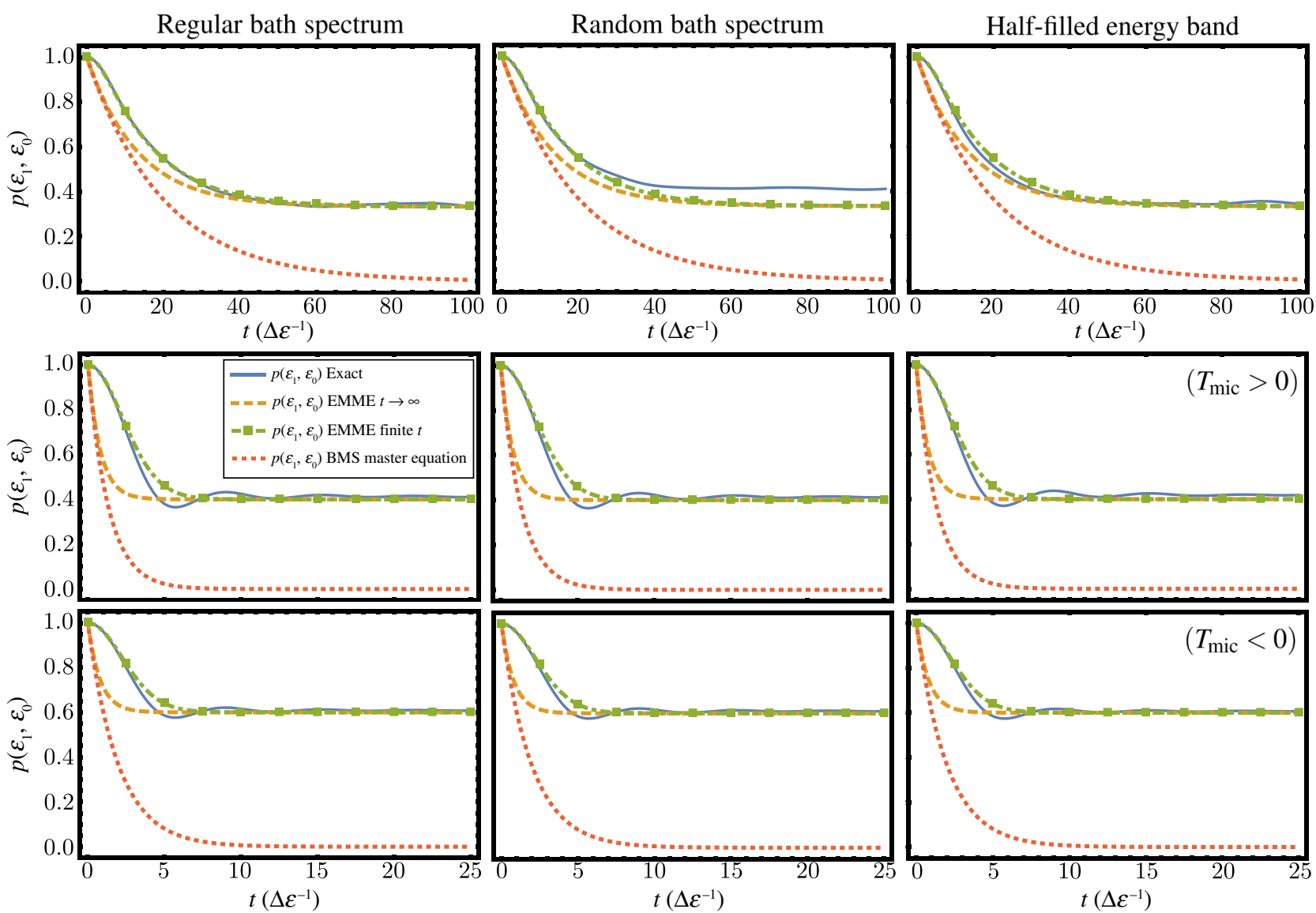

FIG. 9. Comparison of the evolution of the joint probability $p\left(\varepsilon_{1}, E=\varepsilon_{0}\right)$ for a spin system coupled to a two-band bath (see Fig. 2 for details). The first row corresponds to $V_{\varepsilon_{0}}=20$ and $V_{\varepsilon_{1}}=40$. The second row corresponds to $V_{\varepsilon_{0}}=400$ and $V_{\varepsilon_{1}}=600$. The third row corresponds to $V_{\varepsilon_{0}}=600$ and $V_{\varepsilon_{1}}=400 . \lambda=10^{-2}, \delta=0.5, \varepsilon_{0}=0, \varepsilon_{1}=1$, and $a=1$.

The evolution in the interaction picture is then governed by the von Neumann equation

$$
\mathcal{L}(t)[\rho]=-i \sum_{\nu}\left[\tilde{\mathrm{H}}_{\mathrm{int}, v}(t), \tilde{\rho}(t)\right]
$$

Again, it will prove useful to decompose the interaction into the respective block-diagonal and off-diagonal parts

$$
\begin{gathered}
\sum_{\mathbf{E}} \delta \mathrm{H}_{v}(\mathbf{E}) \Pi_{\mathbf{E}}=\lambda \sum_{\alpha_{\nu}} S_{v}^{\alpha_{\nu}} \otimes \sum_{\mathbf{E}}\left\langle B_{\mathrm{int}, \nu}^{\alpha_{\nu}}\right\rangle_{\mathbf{E}} \Pi_{\mathbf{E}}, \\
V_{\nu}=\lambda \sum_{\alpha_{\nu}} S_{v}^{\alpha_{\nu}} \otimes\left(B_{\mathrm{int}, \nu}^{\alpha_{\nu}}-\sum_{\mathbf{E}}\left\langle B_{\mathrm{int}, \nu}^{\alpha_{\nu}}\right\rangle_{\mathbf{E}} \Pi_{\mathbf{E}}\right),
\end{gathered}
$$

and further introduce $\delta \mathrm{H}_{v}=\sum_{\mathbf{E}} \delta \mathrm{H}_{v}(\mathbf{E})$ and $B_{v}^{\alpha_{v}}=B_{\text {int }}^{\alpha_{v}}-$ $\sum_{\mathbf{E}}\left\langle B_{\text {int, } v}^{\alpha_{\nu}}\right\rangle_{\mathbf{E}} \Pi_{\mathbf{E}}$. We now proceed to compute the different terms appearing in Eq. (2). We start with

$$
\mathcal{P} \mathcal{L}(t) \mathcal{P} \rho=-i \sum_{\nu}\left[\delta \tilde{\mathrm{H}}_{\nu}(t), \mathcal{P} \tilde{\rho}(t)\right]
$$

and using the linearity of the commutator together with $\mathcal{Q}=\mathcal{I}-\mathcal{P}$, we deduce

$$
\mathcal{Q L}(t) \mathcal{P} \rho=-i \sum_{\nu}\left[\tilde{V}_{\nu}(t), \mathcal{P} \tilde{\rho}(t)\right]
$$

Therefore, the second-order term is found to be

$$
\begin{aligned}
& \mathcal{P} \mathcal{L}(t) \mathcal{Q} \mathcal{L}\left(t^{\prime}\right) \mathcal{P} \rho \\
& \quad=\sum_{\nu v^{\prime}} \sum_{\mathbf{E}} \operatorname{tr}_{\mathrm{B}}\left(\Pi _ { \mathbf { E } } \left[\delta \tilde{\mathrm{H}}_{\nu}(t)\right.\right. \\
& \left.\left.\quad+\tilde{V}_{\nu}(t),\left[\mathcal{P} \tilde{\rho}\left(t^{\prime}\right), V_{v^{\prime}}\left(t^{\prime}\right)\right]\right]\right) \frac{\Pi_{\mathbf{E}}}{V_{\mathbf{E}}} .
\end{aligned}
$$

After a lengthy but straightforward manipulation, one finds that the term involving $\delta \mathrm{H}_{v}$ vanishes since it ends up being proportional to $\left\langle B_{v}^{\alpha_{v}}\right\rangle_{\mathbf{E}}=0$, and one is left with

$$
\begin{aligned}
& \mathcal{P} \mathcal{L}(t) \mathcal{Q L}\left(t^{\prime}\right) \mathcal{P} \rho \\
& \quad=\sum_{v v^{\prime}} \sum_{\mathbf{E}} \operatorname{tr}_{\mathrm{B}}\left(\Pi_{\mathbf{E}}\left\{\tilde{V}_{v}(t),\left[\mathcal{P} \tilde{\rho}\left(t^{\prime}\right), \tilde{V}_{v^{\prime}}\left(t^{\prime}\right)\right]\right\}\right) \frac{\Pi_{\mathbf{E}}}{V_{\mathbf{E}}} .
\end{aligned}
$$


Using the explicit expression for $V_{v}$, one finds that only the terms with $v=v^{\prime}$ survive since, again, the case $v \neq v^{\prime}$ lead to factors $\left\langle B_{v}^{\alpha_{v}}\right\rangle_{\mathbf{E}}=0$. Therefore, we get an additive structure for the EMME. Then one can proceed for each $v$ as we do for the single bath case. Performing the standard Markov and secular approximations leads to Eq. (71).

\section{Rate equation}

The rate equation for the multiple-environment case is found to be

$$
\begin{aligned}
\partial_{t} p\left(\varepsilon_{k}, \mathbf{E}\right)= & \sum_{v, q}\left(\frac{W_{v, k q}\left(E_{v}, E_{v}+\omega_{k q}\right)}{V_{E_{v}+\omega_{k q}}} p\left(\varepsilon_{q}, \mathbf{E}+\omega_{k q} \hat{\mathbf{e}}_{v}\right)\right. \\
& \left.-\frac{W_{v, q k}\left(E_{v}+\omega_{k q}, E_{v}\right)}{V_{E_{v}}} p\left(\varepsilon_{k}, \mathbf{E}\right)\right),
\end{aligned}
$$

where for each bath $v$ we introduce the transition rates $W_{v, k q}$ analogously to the case of a single bath. Again, we find the properties $W_{v, k q}\left(E_{v}, E_{v}^{\prime}\right) \geq 0$ and $W_{v, k q}\left(E_{v}, E_{v}^{\prime}\right)=$ $W_{v, q k}\left(E_{v}^{\prime}, E_{v}\right)$.

\section{Energy conservation}

Using Eq. (G8), one can prove that $P\left(E_{\text {tot }}\right)=$ $\sum_{k, \mathbf{E}} p\left(\varepsilon_{k}, \mathbf{E}\right) \delta_{E_{\mathrm{tot}}, \varepsilon_{k}+\sum_{v} E_{v}}$ is preserved. To this end we introduce, given $E_{\text {tot }}$, the energy of the bath $v$ given the system energy $\varepsilon_{k}$

$$
E_{v \mid k}=E_{\text {tot }}-\sum_{v^{\prime} \neq v} E_{v^{\prime}}-\varepsilon_{k},
$$

with the property $E_{v \mid k}+\omega_{k q}=E_{v \mid q}$. We note that under the action of the Kronecker $\delta$ function in the definition of $P\left(E_{\text {tot }}\right)$, we have

$$
\begin{array}{rl}
\partial_{t} & P\left(E_{\mathrm{tot}}\right)=\sum_{\mathbf{E}} \sum_{v} \sum_{k q} \\
& \times\left(\frac{W_{v, k q}\left(E_{v \mid k}, E_{v \mid q}\right)}{V_{E_{v \mid q}}} p\left(\varepsilon_{q}, E_{1}, \ldots, E_{v \mid q}, \ldots, E_{n}\right)\right. \\
& \left.-\frac{W_{v, q k}\left(E_{v \mid q}, E_{v \mid k}\right)}{V_{E_{v \mid k}}} p\left(\varepsilon_{k}, E_{1}, \ldots, E_{v \mid k}, \ldots, E_{n}\right)\right),
\end{array}
$$

which vanishes after renaming of the dummy variables $k \leftrightarrow q$ in the second line.

\section{Steady state}

Imposing detailed balance in Eq. (G8), we obtain the steady-state condition

$$
\frac{p\left(\varepsilon_{k}, E_{1}, \ldots, E_{v \mid k}, \ldots, E_{n}\right)}{p\left(\varepsilon_{q}, E_{1}, \ldots, E_{v \mid q}, \ldots, E_{n}\right)}=\frac{V_{E_{v \mid k}}}{V_{E_{v \mid q}}}
$$

which is analogous to the one found for the singleenvironment case in Eq. (35).

[1] H.-P. Breuer and F. Petruccione, The Theory of Open Quantum Systems (Oxford University Press, 2002).

[2] G. Schaller, Open Quantum Systems far From Equilibrium (Springer, 2014), Vol. 881.

[3] I. de Vega and D. Alonso, Dynamics of non-Markovian open quantum systems, Rev. Mod. Phys. 89, 015001 (2017).

[4] M. Esposito and P. Gaspard, Quantum master equation for a system influencing its environment, Phys. Rev. E 68, 066112 (2003).

[5] A. A. Budini, Random Lindblad equations from complex environments, Phys. Rev. E 72, 056106 (2005).

[6] H.-P. Breuer, J. Gemmer, and M. Michel, Non-Markovian quantum dynamics: Correlated projection superoperators and Hilbert space averaging, Phys. Rev. E 73, 016139 (2006).

[7] A. A. Budini, Lindblad rate equations, Phys. Rev. A 74, 053815 (2006).

[8] H.-P. Breuer, Non-Markovian generalization of the Lindblad theory of open quantum systems, Phys. Rev. A 75, 022103 (2007).

[9] M. Esposito and P. Gaspard, Spin relaxation in a complex environment, Phys. Rev. E 68, 066113 (2003).

[10] J. Fischer and H.-P. Breuer, Correlated projection operator approach to non-Markovian dynamics in spin baths, Phys. Rev. A 76, 052119 (2007).

[11] J. von Neumann, Beweis des ergodensatzes und deshtheorems in der neuen mechanik, Z. Phys. 57, 30 (1929).

[12] J. von Neumann, Proof of the ergodic theorem and the Htheorem in quantum mechanics, Eur. Phys. J. H 35, 201 (2010).

[13] P. Reimann, Foundation of Statistical Mechanics under Experimentally Realistic Conditions, Phys. Rev. Lett. 101, 190403 (2008).

[14] L. C. Venuti, The recurrence time in quantum mechanics, arXiv: 1509.04352 (2015).

[15] P. Strasberg, Entropy production as change in observational entropy, arXiv:1906.09933 (2019).

[16] M. Esposito and P. Gaspard, Quantum master equation for the microcanonical ensemble, Phys. Rev. E 76, 041134 (2007).

[17] C. W. J. Beenakker, Random-matrix theory of quantum transport, Rev. Mod. Phys. 69, 731 (1997).

[18] W. M. Gelbart, S. A. Rice, and K. F. Freed, Random matrix theory and the master equation for finite systems, J. Chem. Phys. 57, 4699 (1972).

[19] P. A. Mello, P. Pereyra, and N. Kumar, A soluble randommatrix model for relaxation in quantum systems, J. Stat. Phys. 51, 77 (1988).

[20] P. Pereyra, Random-matrix model for dissipative two-level systems, J. Stat. Phys. 65, 773 (1991).

[21] D. Cohen, F. M. Izrailev, and T. Kottos, Wave Packet Dynamics in Energy Space, Random Matrix Theory, and the Quantum-Classical Correspondence, Phys. Rev. Lett. 84, 2052 (2000).

[22] J. L. Lebowitz and L. Pastur, A random matrix model of relaxation, J. Phys. A 37, 1517 (2004). 
[23] D. Cohen and T. Kottos, Quantum dissipation due to the interaction with chaos, Phys. Rev. E 69, 055201(R) (2004).

[24] C. Nation and D. Porras, Quantum chaotic fluctuationdissipation theorem: Effective Brownian motion in closed quantum systems, Phys. Rev. E 99, 052139 (2019).

[25] P. Reimann, Eigenstate thermalization: Deutsch's approach and beyond, New J. Phys. 17, 055025 (2015).

[26] J. M. Deutsch, Quantum statistical mechanics in a closed system, Phys. Rev. A 43, 2046 (1991).

[27] M. Srednicki, Chaos and quantum thermalization, Phys. Rev. E 50, 888 (1994).

[28] L. D’Alessio, Y. Kafri, A. Polkovnikov, and M. Rigol, From quantum chaos and eigenstate thermalization to statistical mechanics and thermodynamics, Adv. Phys. 65, 239 (2016).

[29] J. M. Deutsch, Eigenstate thermalization hypothesis, Rep. Prog. Phys. 81, 082001 (2018).

[30] C. Maes and K. Netočnỳ, Time-reversal and entropy, J. Stat. Phys. 110, 269 (2003).

[31] S. Braun, J. P. Ronzheimer, M. Schreiber, S. S. Hodgman, T. Rom, I. Bloch, and U. Schneider, Negative absolute temperature for motional degrees of freedom, Science 339, 52 (2013).

[32] V. Romero-Rochín, Nonexistence of equilibrium states at absolute negative temperatures, Phys. Rev. E 88, 022144 (2013).

[33] J. Dunkel and S. Hilbert, Consistent thermostatistics forbids negative absolute temperatures, Nat. Phys. 10, 67 (2014).

[34] M. Campisi, Construction of microcanonical entropy on thermodynamic pillars, Phys. Rev. E 91, 052147 (2015).

[35] E. Abraham and O. Penrose, Physics of negative absolute temperatures, Phys. Rev. E 95, 012125 (2017).

[36] R. H. Swendsen, Thermodynamics of finite systems: a key issues review, Rep. Prog. Phys. 81, 072001 (2018).

[37] U. Schneider, S. Mandt, A. Rapp, S. Braun, H. Weimer, I. Bloch, and A. Rosch, Comment on "Consistent thermostatistics forbids negative absolute temperatures", arXiv:1407.4127 (2014).

[38] M. T. Mitchison and M. B. Plenio, Non-additive dissipation in open quantum networks out of equilibrium, New J. Phys. 20, 033005 (2018).

[39] D. Braun, F. Haake, and W. T. Strunz, Universality of Decoherence, Phys. Rev. Lett. 86, 2913 (2001).

[40] D. Šafránek, J. M. Deutsch, and A. Aguirre, Quantum coarse-grained entropy and thermodynamics, Phys. Rev. A 99, 010101(R) (2019).

[41] D. Šafránek, J. M. Deutsch, and A. Aguirre, Quantum coarse-grained entropy and thermalization in closed systems, Phys. Rev. A 99, 012103 (2019).

[42] J. Schindler, D. Šafránek, and A. Aguirre, arXiv:2005.05408.

[43] P. Strasberg and A. Winter, Entanglement entropy from coarse-graining in pure and mixed multipartite systems, arXiv:2002.08817 (2020).

[44] P. Strasberg and M. Esposito, Non-Markovianity and negative entropy production rates, Phys. Rev. E 99, 012120 (2019).

[45] M. Esposito, U. Harbola, and S. Mukamel, Nonequilibrium fluctuations, fluctuation theorems, and counting statistics in quantum systems, Rev. Mod. Phys. 81, 1665 (2009).

[46] A. Kelly and T. E. Markland, Efficient and accurate surface hopping for long time nonadiabatic quantum dynamics, J. Chem. Phys. 139, 014104 (2013).
[47] A. Kelly, N. Brackbill, and T. E. Markland, Accurate nonadiabatic quantum dynamics on the cheap: Making the most of mean field theory with master equations, J. Chem. Phys. 142, 094110 (2015).

[48] T. Brandes, Coherent and collective quantum optical effects in mesoscopic systems, Phys. Rep. 408, 315 (2005).

[49] D. Segal, Heat flow in nonlinear molecular junctions: Master equation analysis, Phys. Rev. B 73, 205415 (2006).

[50] G. Schaller, T. Krause, T. Brandes, and M. Esposito, Singleelectron transistor strongly coupled to vibrations: counting statistics and fluctuation theorem, New J. Phys. 15, 033032 (2013).

[51] D. Gelbwaser-Klimovsky and A. Aspuru-Guzik, Strongly coupled quantum heat machines, J. Phys. Chem. Lett. 6, 3477 (2015).

[52] C. Wang, J. Ren, and J. Cao, Nonequilibrium energy transfer at nanoscale: A unified theory from weak to strong coupling, Sci. Rep. 5, 11787 (2015).

[53] K. H. Hughes, C. D. Christ, and I. Burghardt, Effectivemode representation of non-Markovian dynamics: A hierarchical approximation of the spectral density. I. Application to single surface dynamics, J. Chem. Phys. 131, 024109 (2009).

[54] R. Martinazzo, B. Vacchini, K. H. Hughes, and I. Burghardt, Communication: Universal Markovian reduction of Brownian particle dynamics, J. Chem. Phys. 134, 011101 (2011).

[55] M. Woods, R. Groux, A. Chin, S. F. Huelga, and M. B. Plenio, Mappings of open quantum systems onto chain representations and Markovian embeddings, J. Math. Phys. 55, 032101 (2014).

[56] K. H. Hughes, C. D. Christ, and I. Burghardt, Effectivemode representation of non-Markovian dynamics: A hierarchical approximation of the spectral density. II. Application to environment-induced nonadiabatic dynamics, J. Chem. Phys. 131, 124108 (2009).

[57] J. Iles-Smith, N. Lambert, and A. Nazir, Environmental dynamics, correlations, and the emergence of noncanonical equilibrium states in open quantum systems, Phys. Rev. A 90, 032114 (2014).

[58] P. Strasberg, G. Schaller, N. Lambert, and T. Brandes, Nonequilibrium thermodynamics in the strong coupling and non-Markovian regime based on a reaction coordinate mapping, New J. Phys. 18, 073007 (2016).

[59] D. Newman, F. Mintert, and A. Nazir, Performance of a quantum heat engine at strong reservoir coupling, Phys. Rev. E 95, 032139 (2017).

[60] P. Strasberg, G. Schaller, T. L. Schmidt, and M. Esposito, Fermionic reaction coordinates and their application to an autonomous Maxwell demon in the strong-coupling regime, Phys. Rev. B 97, 205405 (2018).

[61] D. Tamascelli, A. Smirne, S. F. Huelga, and M. B. Plenio, Nonperturbative Treatment of non-Markovian Dynamics of Open Quantum Systems, Phys. Rev. Lett. 120, 030402 (2018).

[62] M. Brenes, J. J. Mendoza-Arenas, A. Purkayastha, M. T. Mitchison, S. R. Clark, and J. Goold, TensorNetwork Method to Simulate Strongly Interacting Quantum Thermal Machines, Phys. Rev. X 10, 031040 (2020). 